\title{
Young Brown Dwarfs in the Core of the W3 Main Star-Forming Region
}

\author{
D. K. Ojha ${ }^{1}$ \\ Tata Institute of Fundamental Research, Homi Bhabha Road, Colaba, Mumbai 400 005, \\ India \\ and \\ M. Tamura, Y. Nakajima, H. Saito \\ National Astronomical Observatory of Japan, Mitaka, Tokyo 181-8588, Japan \\ and \\ A. K. Pandey \\ Aryabhatta Research Institute of Observational Sciences, Manora Peak, Nainital 263 129, \\ India \\ and \\ S. K. Ghosh \\ Tata Institute of Fundamental Research, Homi Bhabha Road, Colaba, Mumbai 400 005, \\ India \\ and \\ K. Aoki \\ Subaru Telescope, National Astronomical Observatory of Japan, 650 North A'ohoku Place, \\ Hilo, HI 96720
}

\begin{abstract}
We present the results of deep and high-resolution (FWHM $\left.\sim 0^{\prime \prime} .35\right) J H K$ near-infrared observations with the Subaru telescope, to search for very low mass
\end{abstract}

\footnotetext{
${ }^{1}$ E-mail: ojha@tifr.res.in
} 
young stellar objects in the W3 Main star-forming region. The near-infrared survey covers an area of $\sim 2.6 \operatorname{arcmin}^{2}$ with $10 \sigma$ limiting magnitude exceeding 20 mag in the $J H K$ bands. The survey is sensitive enough to provide unprecedented details in W3 IRS 5 and IRS 3a regions and reveals a census of the stellar population down to objects below the hydrogen-burning limit. We construct $J H K$ color-color and $J-H / J$ and $H-K / K$ color-magnitude diagrams to identify very low luminosity young stellar objects and to estimate their masses. Based on these color-color and color-magnitude diagrams, we identified a rich population of embedded YSO candidates with infrared excesses (Class I and Class II), associated with the W3 Main region. A large number of red sources $(H-K>2)$ have also been detected around W3 Main, which are arranged from the northwest toward the southeast regions. Most of these are concentrated around W3 IRS 5. We argue that these red stars are most probably pre-main-sequence (PMS) stars with intrinsic color excesses. We find that the slope of the $K$-band luminosity function of W3 Main is lower than the typical values reported for young embedded clusters. Based on the comparison between theoretical evolutionary models of very lowmass pre-main sequence objects with the observed color-magnitude diagram, we find there exists a substantial substellar population in the observed region. The mass function does not show the presence of cutoff and sharp turnover around the substellar limit, at least at the hydrogen-burning limit. Furthermore, the mass function slope indicates that the number ratio of young brown dwarfs and hydrogen-burning stars in the W3 Main is probably higher than those in Trapezium and IC 348. The presence of mass segregation, in the sense that relatively massive YSOs lie near the cluster center, is seen. The estimated dynamical evolution time indicates that the observed mass segregation in the W3 Main may be the imprint of the star formation process.

Subject headings: infrared: stars - ISM: clouds - ISM: individual (W3) - open clusters and associations: general — stars: formation — stars: pre-main-sequence

\section{Introduction}

One of the most important problems in star formation is understanding the origin of the stellar initial mass function (IMF). The pioneering work by Salpeter (1955) indicated that the IMF in the mass range $0.4-10 \mathrm{M}_{\odot}$ can be presented with power law or lognormal functions. Recent studies have shown a break in the function around $1 \mathrm{M}_{\odot}$ (cf. Kroupa 2001, 2002; Muench et al. 2002, 2003). It is believed that the majority of stars are formed 
in clusters which contain a large number of low mass stars. These low mass stars play an important role in the study of IMF. There are many embedded young clusters in molecular clouds (cf. Lada \& Lada 2003), which are ideal targets for the study of IMF, because the young clusters are known to keep their initial conditions of star formation. However, the molecular clouds have long prevented optical observations of the embedded young clusters, and we had to wait for the development of recent NIR arrays for substantial observations (e.g., Lada \& Lada 2003).

The functional form and the universality of the IMF at a very low mass regime including brown dwarfs (BDs) are still open questions. At the low end of the IMF are BDs which are star-like objects that span the mass range between giant planets like Jupiter and the least massive stars that are just below the hydrogen-burning limit but above the deuteriumburning limit. The expectation that BDs form by the same physical process as low-mass young stars suggests that one might find BDs in the very locations that one finds protostars: molecular clouds. However, it is not clear how abundantly BDs are formed in molecular clouds and whether they significantly contribute to the low-mass end of the luminosity function, and therefore to the IMF. By conducting surveys of the young stellar populations often associated with molecular cloud cores we will be able to characterize the IMF down into the BDs regime and determine: i) if there is a peak in the IMF of young, emerging star clusters near the hydrogen-burning limit (Zinnecker et al. 1993; Muench et al. 2002) and ii) what connection is there, if any, between such a peak and the physical conditions that characterize the molecular cloud. The main difficulty so far is the lack of high spatial resolution and sensitive data in the crowded fields, such as dense cluster cores, where there is a high probability of finding BDs.

So far the young brown dwarf (YBD) search has been limited to the nearby star forming regions (< 500 pc; e.g. Orion, Taurus, Chamaeleon, Lupus, Ophiuchus: Kaifu et al. (2000), Lucas \& Roche (2000), Tamura et al. (1998), Oasa et al. (1999), Nakajima et al. (2000), Comeron et al. (1993), Luhman \& Rieke (1999)). In order to detect and characterize the YBDs in distant massive star forming regions, which are more typical in the galactic scale, we need high sensitivity and high resolution. Because the very low mass stars have infrared excesses when they are young, we can detect YSOs including YBDs only with the colors and magnitudes in the JHK wavelengths (Muench et al. 2001; Liu et al. 2003; Luhman et al. 2007). Since stars are brighter when they are young especially at infrared wavelengths, it is easier to detect very low mass stars in young clusters. Hillenbrand (1997), Hillenbrand \& Carpenter (2000), and Luhman et al. (2000) observed a rich massive star forming region, the Orion star forming region. They obtained results that suggest the slope of IMF changes at $0.2 \mathrm{M}_{\odot}$ and its shape continuously changes towards BD regime. However, the result is derived from only one star forming region. Therefore, it is important to find out if it is 
universal among other distant massive star forming regions.

W3 giant molecular cloud complex is a well-studied star-forming region located in the Perseus arm at a distance of $1.83 \mathrm{kpc}$ that contains objects such as H II regions, embedded IR sources (including the extremely luminous cluster of sources W3 IRS 5), OH and water masers. In order to search for the embedded very low-mass young stellar populations, we have recently analysed a deep $J H K_{\mathrm{s}}$ (simultaneous) survey of W3 Main star-forming region (Ojha et al. 2004a). The observations were carried out with the SIRIUS camera on the UH $2.2 \mathrm{~m}$ telescope. The near-infrared (NIR) survey covers an area of $\sim 24 \operatorname{arcmin}^{2}$ with 10 $\sigma$ limiting magnitudes of $\sim 19.0,18.1$, and 17.3 in $J, H$, and $K_{\mathrm{s}}$ bands, respectively. A significant number of very low-luminosity YSOs have been found in the core region of W3 Main based on their NIR colors (Ojha et al. 2004a), where the estimated stellar density is of $\sim 2000 \mathrm{pc}^{-3}$ (for $K<17.5 \mathrm{mag}$ ). We expect that some of them would be candidates for YBDs recently formed in the W3 molecular cloud.

In this paper we present a new set of $J_{-}, H$-, and $K$-band data for the W3 Main starforming region with higher resolution and sensitivity $(K \sim 20 \mathrm{mag})$ for an area of $\sim 2.6$ $\operatorname{arcmin}^{2}$ centered on W3 IRS 5. Our motivation is to look for the YBDs associated with the W3 Main region and discuss their nature and mass function (MF). In $\S 2$ and $\S 3$ we present the details of the observations and data reduction procedures. Section 4 contains the results and discussion on mostly point-like YSOs and describe the details of substellar population. Section 5 compares the results from previous studies of W3 Main. We then summarize our conclusions in $\S 6$.

\section{Observations}

Deep imaging observations of the W3 Main star-forming region at the NIR wavelengths $J(\lambda=1.25 \mu \mathrm{m}), H(\lambda=1.64 \mu \mathrm{m})$, and $K(\lambda=2.21 \mu \mathrm{m})$ were obtained on 2004 August 27, using the facility instrument Cooled Infrared Spectrograph and Camera for OHS (CISCO) mounted on the Cassegrain focus of the Subaru $8.2 \mathrm{~m}$ telescope. CISCO is equipped with a $1024 \times 1024$ Rockwell HgCdTe HAWAII array. A plate scale of $0^{\prime \prime} .105$ pixel $^{-1}$ at the $\mathrm{f} / 12$ focus of the telescope provides a field-of-view of $\sim 1^{\prime} .8 \times 1^{\prime} .8$ (Motohara et al. 2002). The off-target sky frames located at $\sim 30^{\prime}$ north of the target position were also taken just after the object frames. The sky frame was also used as a reference field for W3 Main to assess the stellar populations within the W3 Main star-forming region (see $\S 4$ ). The exposure times for individual frames were 40,20 , and $20 \mathrm{~s}$ in $J, H$, and $K$, respectively, yielding a total integration time of 12 minutes in all three bands. Each position was observed in a $3 \times 3$ dithered pattern with $10^{\prime \prime}$ offset, and three to six images were obtained at each dithered 
position. All the observations were done under excellent photometric sky conditions. The average seeing size was measured to be $0^{\prime \prime} .35 \mathrm{FWHM}$ in all three filters. The air mass varied only between 1.09 and 1.38. The United Kingdom Infrared Telescope faint standard star, FS6 (Hawarden et al. 2001), was observed at air mass values close to the target observations, for the photometric calibration.

\section{Data Reduction}

Data reduction was done using the Image Reduction and Analysis Facility (IRAF) software package1 1 . The sky flats were generated by median-combining sky frames of the individual dithering sequences for each filter. The flat-fielding and sky subtraction with a median sky frame were applied. Identification and photometry of point sources were performed by using the DAOFIND and DAOPHOT packages in IRAF, respectively. Because of source confusion and nebulosity within the region, photometry was obtained using the point spread function (PSF) algorithm ALLSTAR in the DAOPHOT package (Stetson 1987). We used an aperture radius of 4 pixels $\left(\sim 0^{\prime \prime} .4\right)$ with appropriate aperture corrections per band for the final photometry. We evaluated photometric accuracy by direct comparison of our results with SIRIUS photometry (Ojha et al. 2004a). We selected point sources that are measured in all three bands with errors $<0.1 \mathrm{mag}$. Comparing between our photometry and SIRIUS photometry, the sources with $J-H<2$ have photometric dispersions of $\sim 0.10$ mag in $J H K$ bands. The dispersion increases to $\sim 0.15 \mathrm{mag}$ for the sources with redder colors $(J-H>2)$. Our higher spatial resolution permits better source separation and sky determination. We have assumed that the resulting $J, H$, and $K$ magnitudes are the same between CIT system and the SUBARU/CISCO system. Absolute position calibration was achieved using the coordinates of a number of stars from the SIRIUS photometric data by Ojha et al. (2004a). The position accuracy is better than $\pm 0^{\prime \prime} .04 \mathrm{rms}$ in the W3 Main field.

The completeness limits of the images were evaluated by adding artificial stars of different magnitudes to the images and determining the fraction of these stars recovered in each magnitude bin. The recovery rate was greater than $90 \%$ for magnitudes brighter than 20.7, 19.0, and 18.5 in the $J, H$, and $K$ bands, respectively. The observations are complete (100\%) to the level of $18.5,17.5$, and $17.0 \mathrm{mag}$ in the $J, H$, and $K$ bands, respectively. The $10 \sigma$ limiting magnitudes for our observations are estimated to be $\sim 22,21$, and 20 in the $J_{-}, H$-, and $K$-bands, respectively.

\footnotetext{
${ }^{1}$ IRAF is distributed by the National Optical Astronomy Observatory, which is operated by the Association of Universities for Research in Astronomy, Inc., under contract to the National Science Foundation.
} 
We also assessed the photometric incompleteness of our sample around the central W3 IRS 5 cluster and diffuse region to the northeast (shown by a box drawn with the dashed lines in Figure 1) and compared it with the incompleteness for the whole region. We do not find significant difference in the completeness ratio as a function of magnitudes between the two regions. The completeness ratios for the cluster and northeast region are $\sim 10 \%$ less as compared to the whole region at fainter magnitudes. We therefore applied an averaged completeness factor across the field for further analysis, as it will not affect our qualitative results.

\section{Results and Discussion}

\subsection{Morphology}

The $J_{-}, H$-, and $K$-band images of the W3 Main star-forming region are shown in Figure 1. A cluster of young stellar objects is seen around the W3 IRS 5 source, which has been detected only in the $K$ band. The individual compact H II regions, ultracompact H II regions, and embedded IR sources are marked in the $K$-band image. The density enhancement is seen around IRS 5 molecular clump in the $K$-band. A composite color image was contructed from the CISCO $J_{-}, H$-, and $K$-band images ( $J$ represented in blue, $H$ in green, and $K$ in red) and is shown in Figure 2. This image is the highest resolution NIR image of the region from the ground and probably the deepest to date. Our deep and high-resolution NIR images show distinctive reddish and bluish nebulosity features, dark filaments between the diffuse nebulosity, and a significant population of faint stars in W3 Main. A large number of red young stars that are presumably embedded in the molecular core are also seen around IRS 5 and IRS 3a. The overall morphology and sizes of the bright nebulosities are quite similar to those in Hubble Space Telescope (HST) NIR images within $\sim 1^{\prime} .0 \times 1^{\prime} .0$ (Megeath et al. 2005). However, the comparison of the K-band images (see Appendix and Fig. 3 in Megeath et al. 2005) shows that the Subaru images looks a little deeper than the HST images, in spite of the their slightly better resolution. Notable nebulosity features are summarized in Appendix.

\subsection{Photometric Analysis of Pointlike Sources}

We obtained photometric data of 295 sources within a $2.6 \operatorname{arcmin}^{2}$ area in $J H K$ bands with photometric errors in each band of less than 0.1 mag. Megeath et al. (2005) presented HST NICMOS imaging of W3 IRS 5, a binary high-mass protostar. They showed three new 
$2.22 \mu \mathrm{m}$ sources (NIR 3 - 5) with very red colors in addition to the two protostars (NIR 1 and NIR 2). NIR 1 and NIR 2 are saturated in the Subaru $K$-band image, whereas the three new sources (NIR $3-5$ ) are clearly resolved. In addition to this we find an additional source about $0^{\prime \prime} .8$ southwest of NIR 4 . We designate this source as NIR 6. While comparing with the photometry, we find that NIR 6 is probably not an independent source but is most likely an unresolved gaseous knot in the nebula (see Appendix and related Figure 14a for a more detailed discussion of the W3 IRS 5 system).

We compared our derived photometry for the NIR 3 - 5 sources to those in Megeath et al. (2005). The agreement of magnitudes is encouraging except for IRS 3 which is a young OB star embedded in the molecular core (Megeath et al. 2005). It is likely that NIR 3 has brightened by $\sim 0.6$ mag within a period of above six years $(1998-2004)$. In addition to NIR 1 - 6, we see several red sources and a detailed structure of the IR nebula just to the north of NIR 1 in our deep $K$-band image (see Appendix).

\subsubsection{Color-Color Diagram}

Figure 3 shows $J H K$ color-color (CC) diagrams of the W3 Main star-forming region and the reference field, respectively, for the sources detected in the $J H K$ bands with photometric errors in each color of less than $0.1 \mathrm{mag}$. The reference field is also used for correction of field star contamination in the raw KLF of W3 Main (see §4.4). Due to heavy extinction, only a limited number of sources have been detected in $J$ band. The solid thin curve represents the locus of main-sequence (MS) and thick-dashed curve is for giant stars taken from Bessell \& Brett (1988). The dotted line represents the classical T Tauri (CTT) locus as determined by Meyer et al. (1997). Presuming universal nature of the extinction law in NIR region (Mathis 1990), we plotted reddening vectors of the normal reddening law as parallel dashed lines. We assumed that $A_{J} / A_{V}=0.265, A_{H} / A_{V}=0.155, A_{K} / A_{V}=0.090$ for CIT system (Cohen et al. 1981). As can be seen in Figure 3, the stars in W3 Main are distributed in a much wider range than those in the reference field, which indicates that a large fraction of the observed sources in W3 Main exhibit NIR excess emission characteristics of young stars with circumstellar material, as well as a wide range of reddening. We have classified the CC diagram into three regions (e.g., Sugitani et al. 2002; Ojha et al. 2004a, 2004b) to study the nature of sources. The "F" sources are located within the reddening band of the MS and giant stars. These stars are generally considered to be either field stars, Class III objects, or Class II objects with small NIR excess. "T" sources are located redward of region F, but blueward of the reddening line projected from the red end of the CTT locus of Meyer et al. (1997). These sources are considered to be mostly T Tauri stars (Class II objects) with large 
NIR excess. "P" sources are those located in the region redward of region $\mathrm{T}$, and are most likely Class I objects (protostellar objects). There may be an overlap in the NIR colors of the upper end band of Herbig Ae/Be stars and in the lower end band of T Tauri stars in region $\mathrm{T}$ (Hillenbrand et al. 1992). The majority of sources in our sample are distributed in the F region. The total number of "T" (Class II) and "P" (Class I) sources are 89 and 78, respectively. However, this is the lower limit, as several cluster members detected in the $K$ band may not be detected in the other two shorter NIR wavelength bands.

\subsubsection{Color-Magnitude Diagram}

Figure 4 (left) shows a $H-K$ versus $K$ color-magnitude (CM) diagram of the W3 Main star-forming region, where all the sources detected in the $J H K$ bands plus some 160 stars fainter than our limit at the $J$ band but still above the detection threshold in the $H$ and $K$ bands are plotted. The vertical solid lines represent zero-age-main-sequence (ZAMS) curves (for a distance of $1.83 \mathrm{kpc}$ ) reddened by $A_{V}=0,15,30,45$, and $60 \mathrm{mag}$, respectively. The parallel slanting lines represent the reddening vectors to the corresponding spectral type. An apparent MS track is noticeable at $H-K \sim 0.4$ in this diagram; however, a comparison of it with a similar diagram for the stars in the control field (Fig. 4; right) shows that it is a sequence caused by field stars in the foreground of W3 Main. It also appears that the reference field has foreground extinction of the order of $3-5$ visual magnitudes. A comparison of the $J-H$ versus $H-K \mathrm{CC}$ and $H-K$ versus $K \mathrm{CM}$ diagrams of the reference field and W3 Main region indicates that stars having $H-K<1.0$ are probable foreground sources. In Figure 4 (left), YSOs (Classes II and I) found from the CC diagram (Fig. 3) are shown as stars and triangles, respectively. However, it is important to note that even those sources not shown with stars or triangles may also be YSOs with an intrinsic

color excess, since some of them are detected in the $H$ and $K$ bands only and are not in the $J$ band because of their very red colors. Figure 4 (right) shows that there is a lack of sources brighter than about $K=14.5$ in the reference field, which are saturated in our observations. We have complemented the control field observations with 2MASS sources which are shown with cross symbols. The number of such bright stars in our field-of-view is small and does not contribute the object field as extincted background stars that mimic YSOs.

\subsection{Spatial Distribution of Young Stellar Sources}

In our deep NIR observations, 130 very red sources are detected in the $H$ and $K$ bands, out of which only 10 sources have $J$ counterpart. The sources having colors redder than 
$H-K>2$ in Figure 4 (left) are also considered as YSO candidates. In Figure 5 the spatial distribution of the YSO candidates identified in Figures 3 and 4 is shown. Stars represent sources of T Tauri type (Class II), filled triangles indicate Class I sources, and filled circles denote the very red sources $(H-K>2)$. The white contours in Figure 5 represent ${ }^{12} \mathrm{CO}(1-$ 0 ) emission obtained with Nobeyama Radio Observatory $45 \mathrm{~m}$ telescope (T. Sakai, private communication).

In general, Class I and Class II candidates are distributed all over the field; however, an apparent concentration of Class I sources (filled triangles) around W3A, W3B, and $30^{\prime \prime}$ southeast of W3A H II regions can be noticed in Figure 5. It appears that most of these YSOs (Class I sources) are associated with the diffuse ionized gas at the edge of these H II regions. Most of the sources with large color indices $(H-K>2)$ also seem to be distributed all over the field, barring the W3A H II region. However, an apparent concentration of these sources can be seen around W3F, IRS 7 and IRS 5 regions (see Figs. 1 \& 5). The lack of these red sources around W3A H II region indicates that this region is relatively evolved as compared to other regions.

If we assume that the large $H-K(>2)$ color results merely from the interstellar reddening affecting normal stars, then the extinction value might even exceed 60 mag in the molecular cloud in which some of the red stars are found (see Fig. 4). However, with such a large amount of absorption, diffuse emissions are unlikely to be detected in the NIR. Since most of the red sources are associated with faint diffuse emission across the field (Fig. 5), this provides evidence that these sources are YSOs with intrinsic NIR excesses and possibly local extinction as well. We also find that the mean $A_{V}$ value of the Class II sources around W3 IRS 5 dense cluster region is about 21 mag. A similar value of $A_{V}$ for the red sources indicates that large $H-K$ color of these sources should be due to NIR excess. This further provides evidence that the red sources should be PMS objects still embedded in clouds.

\subsection{The $K$-band Luminosity Function}

The $K$-band luminosity function (KLF) of an embedded cluster is useful in constraining the age of the cluster (Megeath et al 1996; Ojha et al 2004a). Our motivation is also to compare the KLF slope obtained from deeper Subaru $K$-band data with the results from previous studies of W3 Main (Ojha et al. 2004a; Megeath et al. 1996). In order to derive the observed KLF, one needs to apply corrections for (1) the incompleteness of the star counts as a function of the $K$ magnitude, and (2) the field star contribution in the line of sight of the cluster. 
We have determined the completeness of our $K$-band data through artificial star experiments using ADDSTAR in IRAF (see §3). This was performed by adding fake stars in random positions into the images at 0.5 magnitude intervals and then by checking how many of the added stars could be recovered at various magnitude intervals. We repeated this procedure at least five times. We thus obtained the detection rate as a function of magnitude, which is defined as the ratio of the number of recovered artificial stars over the number of added stars.

One method to correct for the foreground and background contamination is to use a reference field, located reasonably away from the cluster, and yet close enough to have a similar Galactic field star distribution, e.g., with the same Galactic latitude. The observed reference field is then assumed to represent the contaminating field population in the W3 Main region. However, in the case of a young and partly embedded region like W3 Main, the colors and magnitudes of the background contaminating populations are significantly affected by the parent molecular cloud. To correct for the foreground and background contamination, we used of both the Galactic model by Robin et al. (2003) and the reference field star counts. This Galactic model of population synthesis reproduces the stellar contents of the Galaxy. The star counts were predicted using the Besançon model of stellar population synthesis (Robin et al. 2003) in the direction of the reference field close to the W3 Main (see §2), which is also corrected for photometric completeness. To ensure that the model does a reasonably good job in predicting the field star population, we compared the model star counts with those of our observed reference field. Figure 6 shows the comparison of the completeness corrected KLF of the reference field with the model star counts where all the stars are made fainter by an interstellar extinction of $A_{V}=5$ mag. It can be seen that the model star counts match reasonably well with the observed reference field. Hence, we used this model to predict the contamination to the W3 Main field, for which all the field stars are made fainter by an interstellar extinction of $A_{V}=5 \mathrm{mag}$, whereas the background stars are made fainter by an additional cloud extinction.

The advantage in using this model is that the background stars $(d>1.83 \mathrm{kpc})$ can be separated from the foreground stars $(d<1.83 \mathrm{kpc})$. While all the stars in the field suffer a general interstellar extinction, only the background stars suffer an additional extinction due to the molecular cloud. From Figure 4 we conclude that the average extinction to the embedded cluster is $A_{V} \sim 15 \mathrm{mag}(H-K \sim 1.0)$. Assuming spherical geometry, background stars are seen through $A_{V}=30 \mathrm{mag}(2 \times 15 \mathrm{mag})$. Therefore, we applied an extinction value of $A_{V}=30 \mathrm{mag}$ ( or $A_{K}=2.7 \mathrm{mag}$ ) in simulating the background stars. We combined the foreground and background stars to make a whole set of the contamination field and obtained the fraction of the contaminating stars over the total model counts. Then we scaled the model prediction to the star counts in the reference field, and subtracted the 
combined foreground $(d<1.83 \mathrm{kpc})$ and background $\left(d>1.83 \mathrm{kpc}\right.$ with $\left.A_{K}=2.7 \mathrm{mag}\right)$ data from the KLF of the W3 Main region.

After correcting for the foreground and background star contamination and photometric completeness, the resulting KLF is presented in Figure 7 for the W3 Main region, which follows a power-law in shape. In Figure 7, a power-law with a slope $\alpha\left[d N\left(m_{K}\right) / d m_{K} \propto\right.$ $10^{\alpha m_{K}}$, where $N\left(m_{K}\right)$ is the number of stars brighter than $\left.m_{K}\right]$ has been fitted to the KLF using a linear least-squares fitting routine. The KLF of the W3 Main region shows a powerlaw slope of $\alpha=0.14 \pm 0.03$ over the magnitude range 14 - 18 in the $K$-band. The derived KLF slope is lower than those generally reported for the young embedded clusters $(\alpha \sim 0.4$, e.g., Lada et al. 1991, 1993; Lada \& Lada 2003). Thus, this low value of the slope is indeed an intrinsic property of the stellar population in the W3 IRS 5 region.

\subsection{Age and Stellar Mass Estimates}

The fraction of NIR excess stars in a cluster is an age indicator because the disks/envelopes become optically thin with the increasing age. For young embedded clusters having age $\sim 1 \times 10^{6} \mathrm{yr}$, the fraction of NIR excess stars (based on $J H K$ ) is found to be $\sim 50 \%$ (Lada et al. 2000; Haisch et al. 2000) to $\sim 65 \%$ (Muench et al. 2001). The fraction, in the case of Taurus dark clouds (age $\sim 1-2 \times 10^{6} \mathrm{yr}$ ) is estimated as $40 \%$ (Kenyon \& Hartmann 1995), which decreases to $\sim 20 \%$ for the clusters with age $\sim 2-3 \times 10^{6}$ yr (Haisch et al. 2001; Teixeira et al. 2004; Oliveira et al. 2005).

To estimate the fraction of the NIR excess stars in our sample, we have to estimate the contamination in W3 Main region due to field stars. In order to estimate the foreground and background contaminations, we made use of both the observed star counts and the Galactic model (see more details in §4.4). Using the model predictions and the reference star counts, we obtained a fraction of about $17 \%$ foreground and background contaminating stars in our sample. After correcting for the photometric completeness, foreground and background star contamination, the fraction of the NIR excess stars can be estimated to be $65 \%$, which suggests an upper age limit of $\sim 1 \times 10^{6}$ yr for the W3 Main region. However, to further improve the statistics, observations in $L$ and $M$-bands are needed.

In their NIR survey of the central region $\left(\sim 1^{\prime} .5 \times 1^{\prime} .5\right)$ around W3 IRS 5 , Megeath et al. (1996) found that about $30 \%$ of the NIR sources were Class I objects and concluded that the age of the low mass population is $>0.5 \mathrm{Myr}$. Present data also indicate that about $34 \%$ YSOs are the Class I sources. On the basis of HST NICMOS imaging of W3 IRS 5 region, Megeath et al. (2005) proposed a nascent Trapezium system in the center of the W3 IRS 
5 cluster. On the basis of above discussions we assume an age of 1 Myr for the W3 IRS 5 cluster region.

We estimated the extragalactic contamination in the NIR excess sources of our sample (see also Oasa et al. 2006) by assessing the number of extincted galaxies (or faint background galaxies) using an average number of galaxy counts (Gardner et al. 1993; McLeod et al. 1995; Saracco et al. 1999; Maihara et al. 2001). We assume an average extinction of $A_{V}=30$ mag (or $A_{K}=2.7 \mathrm{mag}$; see $\S 4.4$ ) due to the W3 Main region. The total number of galaxies in our observed region, i.e. $\sim 2.6 \mathrm{arcmin}^{2}$, is predicted to be $\sim 3$ down to our $10 \sigma$ limiting extincted magnitude of 20 in the $K$-band. We therefore conclude that the NIR excess sources in our sample are most likely YSOs associated with the molecular cloud.

The mass of the probable YSO candidates can be estimated by comparing their locations on the CM diagram with evolutionary models of PMS stars. To minimize the effect of the "excess" emission in the NIR, we used $(J-H)$ versus $J$ CM diagram instead of the $(H-K)$ versus $K \mathrm{CM}$ diagram to derive the ages and masses of the YSOs. Figure 8 represents $(J-H)$ versus $J$ CM diagram for probable YSOs (Classes II and I). The symbols are same as in Figure 4. The solid curve in Figure 8 denotes the loci of 1 Myr old PMS stars from Palla \& Stahler (1999) over the mass range 1.4 - $4.0 M_{\odot}$, at an assumed distance of 1.83 kpc. The dotted curve represents a PMS isochrone of 1 Myr from Baraffe et al. (1998; $2003)$ for low-mass YSOs $\left(0.005-1.4 M_{\odot}\right)$. The solid and dotted oblique reddening lines denote the positions of PMS stars of 2.0, 0.1, 0.02, and $0.01 M_{\odot}$ for $1 \mathrm{Myr}$. Most of the objects well above the PMS tracks are luminous and massive ZAMS stars (see Table 1 in

Ojha et al. 2004a). As can be seen in Figure 8, the majority of YSOs have masses in the range of $0.01-2.0 M_{\odot}$, indicating a significant population of very low mass stars below the hydrogen-burning limit.

\subsection{Mass Function}

The interpretation of the KLF as IMF is complicated because of the NIR excess arising from the circumstellar disks. We therefore prefer $J$-band luminosity function (JLF) to determine the MF of the YSOs (Class II \& Class I), identified using their NIR excess properties, which is least affected by the circumstellar matter compared to what is expected solely from the stellar photosphere. We assume that all the YSOs are members of the W3 Main field and the field contamination is negligible in the line of sight of the cluster (see Fig. $4 \& \S 4.2 .2$ ). We constructed reddening-corrected JLF for the Class II sources (Fig. 9; left). Their luminosities are corrected for individual extinction determined by dereddening to the reddening-free locus of classical T Tauri stars or its extension in $(H-K)$ versus $(J-H)$ CC diagram (Fig. 
3) as determined by Meyer et al. (1997). The JLF was also corrected for completeness which was evaluated by using artificial star experiment (see §3). We also constructed JLF for both the Class II and Class I sources, although Class I objects are much younger and therefore more luminous than Class II objects. We assumed an average extinction (derived from the individual extinction estimates for the identified Class II objects) for all the Class I objects and dereddened these objects with this average extinction value. Figure 9 (right) presents the JLF for Class II and Class I sources. The completeness limits used in the JLFs (Fig. 9 ) are determined from the dereddening of the $J$-band completeness limits (see $\S 3$ ) by an average extinction. By looking at the star - brown dwarf (hereafter star-BD) boundary at $1 \mathrm{Myr}$ in Figure 9, there appears to be a substantial substellar population in W3 Main and YBDs can thus be distinguished from low mass $\left(\geq 0.1 M_{\odot}\right)$ YSOs in this JLF.

We derived a relation between $J$-band luminosity and mass for evolutionary models of very low mass objects from Baraffe et al. (1998), Baraffe et al. (2003), and Palla \& Stahler (1999). The Baraffe et al.'s models are designed for very low mass objects, including giant planets, while the Palla \& Stahler (1999) model covers a relatively intermediate mass range. Figure 10 shows the derived mass - $J$-band luminosity relationship for the $1 \mathrm{Myr}$ age based on the theoretical models of Baraffe et al. (1998; 2003), and Palla \& Stahler (1999). We find that the derived isochrones are in reasonable agreement for $\geq 0.01 M_{\odot}$. Using this mass-luminosity relation, the star-BD boundary $\left(0.075 M_{\odot}\right)$ at $1 \mathrm{Myr}$ corresponds to $J_{0} \sim$ 17.75 mag.

The JLF was converted to MF using the mass - $J$-band luminosity relation. The MF of Class II objects was obtained by counting the number of stars in various mass bins and is shown in Figure 11 (left). We have constructed MF in wide bins in order to minimize uncertainties in the mass estimate. Figure 11 (right) also presents the composite MF for Class II and Class I sources. Overall, the shape of the composite MF looks similar to the Class II sources (Fig. 11), but with a different slope.

The MF of W3 Main (Fig. 11) appears to rise monotonically across the hydrogenburning limit up to $\sim 0.04 M_{\odot}$ and seems to have resemblance with that of the S106 (Oasa et al. 2006), whereas the MF for the center core region in the ONC with the detection limit $\sim 0.02 M_{\odot}$, is characterized by a local peak near $0.2-0.3 M_{\odot}$ and appears to fall into the BD regime (Lucas \& Roche 2000; Hillenbrand \& Carpenter 2000). A similar trend is seen by Muench et al. (2002) in their study of MF in the Trapezium cluster. It is to be noted that at $\sim 0.1 M_{\odot}$, the completeness factor of our $J$-band data is about $84 \%$ and it falls to $50 \%$ around $0.05 M_{\odot}$. The MFs have slopes $(d \log (N) / d \log (M))$ of $-0.23 \pm 0.02$ and -0.66 \pm 0.04 , respectively, for the Class II and composite samples, over the mass range $0.04<$ $M / M_{\odot}<1.6$. Here, it is worthwhile to mention that Class I sources have higher NIR excess, 
therefore the mass estimates of these objects on the basis of JLF may be biased towards higher values. If it is true, the slope of the MF for Class I objects will be steeper. We assess the issue of the increase of dispersion at fainter $J$ magnitudes which affects the de-reddening and hence MFs. We find that the dispersion is small enough to consider it unimportant as it does not change the overall shape of the MFs and therefore the qualitative conclusions as drawn above are not affected.

We also estimated the MFs derived using an average extinction for both the Class II and composite samples. The completeness-corrected MFs are shown as continuous thin lines in Figure 11. The overall shapes of the MFs look similar and still indicate a monotonic rise across the hydrogen-burning limit up to $\sim 0.04 M_{\odot}$. The qualitative results do not affect the conclusions as drawn above. However, the slopes of MFs have changed to $-0.74 \pm 0.11$ and $-1.00 \pm 0.08$, respectively, for the Class II and composite samples, over the mass range $0.04<M / M_{\odot}<1.6$.

We also checked the distribution of MF by excluding the sources in the "P" region that fall below $J-H=1.0$. It may be possible that sources fall in this region of the diagram because of photometric defects. Figure 12 (left) presents the MF for the Class I and Class II sources by removing those sources that fall below $J-H=1.0$ in the "P" region. Since there are very few such sources (12 sources) in our sample, the overall statistics do not change. The overall shape of the MF does not change and hence the conclusions drawn above are not affected. We further checked the effect of $A_{V}$ on the MF with the help of extinction distribution in the W3 Main region. This technique has been used in young galactic clusters which have variable amount of reddening (e.g. Pandey et al. 2008). To map the extinction in the W3 Main region we used sources lying in the " $F$ " region, which show a range in the $A_{V}$ from 0 to $35 \mathrm{mag}$ (see Fig. 3). To estimate the $A_{V}$, we traced the stars in "F" region in the reddening band to the solid line shown in the Figure 3, near the MS locus (M0 M6). The whole region was divided into small cells of $200 \times 200$ pixels $\left(\sim 0^{\prime} .35 \times 0^{\prime} .35\right)$ so that each cell has significant number of stars. The mean $A_{V}$ value of each cell was used to deredden the Class II ("T" region) and Class I ("P" region) sources lying in that cell. Figure 12 (right) shows the composite MF for all the Class II and Class I sources lying in "T" and "P" regions. The completeness-corrected MF as shown by a dashed-dotted line in Figure 12 (right) still indicates a monotonic rise across the hydrogen-burning limit and the qualitative results do not affect the conclusions drawn in this section.

Comparison of MF of young star forming regions provides an important diagnostic to study the evolution of star formation process in these regions. Comparison can reveal similarities or differences in MFs that would test the universality of the MF. Comparison of the MF of the W3 Main and that of S106 by Oasa et al. (2006) reveals resemblance, in the 
sense that there is no break in MF slope in BD regime. Whereas the MF for Trapezium and IC 348 clusters declines below $0.12 M_{\odot}$ and $0.08 M_{\odot}$, respectively (Muench et al. 2002; 2003). The derived MF slopes indicate that the number ratio of YBDs and hydrogen-burning stars in the W3 Main is probably higher than those in the ONC and its Trapezium cluster.

\subsection{Mass Segregation and the Formation Scenario of YBDs in W3 Main}

The distribution of YSOs can be used to probe the star formation history of the region. Figure 13 shows the spatial distribution of Class II objects, on the $K$-band image. In this Figure the blue circles show the sources with $J_{0}<17.25 \mathrm{mag}\left(M>0.1 M_{\odot}\right)$ and the red ones are those with $J_{0}>17.25 \mathrm{mag}\left(M<0.1 M_{\odot}\right)$. The separation at $J_{0}=17.25 \mathrm{mag}(M$ $\left.\sim 0.1 M_{\odot}\right)$ is made as it allows us to distinguish relatively massive YSOs $\left(J_{0}<17.25 \mathrm{mag}\right)$ from possible YBDs $\left(J_{0}>17.25 \mathrm{mag}\right)$ in our sample. It is noteworthy to see that most of the brighter ones $\left(J_{0}<17.25 \mathrm{mag}\right.$; relatively massive YSOs) are concentrated at the center of the image around W3 IRS 5 and W3 IRS 3a regions, whereas the fainter ones $\left(J_{0}>17.25\right.$ mag; possible YBD candidates) are away from the dense molecular regions. The distribution of other point sources appears to be quite uniform for both the $J_{0}$ mag ranges.

This result can be interpreted as follows: 1) This is a kind of mass segregation where massive stars tend to stay in the cluster center while low-mass stars are further away. To characterize further the degree of mass segregation in W3 Main we show the distribution of Class II sources, combined distribution of Class II + Class I sources, and all the sources lying in " $\mathrm{F}$ " region of the $(J-H)$ versus $(H-K) \mathrm{CM}$ diagram (Fig. 3), as a function of radius in two different magnitude groups in Figure 14. The radial distance is calculated with respect to the coordinates of W3 IRS 5. The figure clearly indicates an effect of mass segregation in the radial distribution of Class II sources in the sense that the relatively massive YSOs $\left(\mathrm{J}_{0}\right.$ $\left.<17.25 ; M>0.1 M_{\odot}\right)$ tend to lie toward the cluster center, whereas possible $\mathrm{YBDs}_{\left(\mathrm{J}_{0}>\right.}$ $\left.17.25 ; M<0.1 M_{\odot}\right)$ are found away from the center. The segregation is less prominent in the case of combined distribution of Class I and Class II objects. Since we have applied a mean value of $A_{V}$ (see $\left.\S 4.6\right)$ to calculate the $J_{0}$ for Class I sources, this could be a probable cause for the dilution of segregation effect. The "F" region sources do not show any segregation; although some patchy extinction just around W3 IRS 5 provides an impression of a paucity of the "F" region sources near IRS 5, the radial averaged $A_{V}$ is quite smooth out up to 0.6 pc. Similar mass segregation has been reported in several star-forming regions, e.g., ONC, IC 348, S106 (cf. Oasa et al. 2006) as well as in young star clusters (e.g., Pandey et al. 2005; Sharma et al. 2007; Jose et al. 2008).

Dynamical effects could be the probable cause of the mass segregation. Because of 
the dynamical relaxation, low mass stars in a cluster may acquire larger random velocities, consequently occupy a larger volume than high mass stars (cf. Mathieu 1985; Mathieu \& Latham 1986; McNamara \& Sekiguchi 1986). We estimated the relaxation time to decide whether the mass segregation discussed above is primordial or due to dynamical relaxation. To estimate the dynamical relaxation time $T_{E}$, we used the relation

$$
T_{E}=\frac{8.9 \times 10^{5} N^{1 / 2} R_{h}^{3 / 2}}{\bar{m}^{1 / 2} \log (0.4 N)}
$$

where $\mathrm{N}$ is the number of cluster stars, $R_{h}$ is the radius containing half of the cluster mass and $\bar{m}$ is the average mass of cluster stars (Spitzer \& Hart 1971). The lower limit of the stellar mass associated with the W3 IRS 5 system was estimated by Megeath et al. (2005) as about $54 M_{\odot}$. The total number of probable members of the cluster are estimated to be 170 (3 main-sequence stars, 89 Class II objects, and 78 Class I objects). Fifty percent of the probable members are found to be located within $0.6 \operatorname{arcmin}(\sim 0.32 \mathrm{pc})$ of IRS 5 . We assumed this value as the half-mass radius of the cluster. Using these numbers, the estimated relaxation time $T_{E}$ comes out to be $\sim 2 \mathrm{Myr}$, which, within uncertainity, is comparable to the age of the PMS stars of W3 Main. Here it is interesting to mention that Feigelson \& Townsley (2008) have found that some of the OB stars in the core of W3 Main are formed after the bulk of the more widely distributed cluster PMS stars. This form of age spread has long been noted in star clusters (e.g. Herbst \& Miller 1982; DeGioia-Eastwood et al. 2001; Pandey et al. 2005, 2008; Sharma et al. 2007; Jose et al., 2008). If star formation is still going on in the region, the W3 Main OB stars confirms the extreme youth of the cluster, which consequently indicates that the observed mass segregation in the region may be of primordial nature.

2) Similar to 1) but alternatively, it might reflect the formation scenario for brown dwarfs. If the ejection scenario for brown dwarf formation is correct, we can also expect the same effect (i.e., BDs tend to go away from the cluster center after their formation) and finally 3 ) it could also be due to some observation bias. The sensitivity of lowest mass objects could be slightly lower due to the higher stellar density toward the center or due to their associated nebulosity. This could be a problem in S106 where the nebula is brightest near the center of the system. But at least the nebulosity around W3 IRS 5 is not a problem because we have detected YBDs even in the bright nebulosity around W3 A (Fig. 13). 


\section{Comparison With Previous Studies}

W3 Main region has been studied in detail at radio, millimeter, and infrared wavelengths, however, little is known about its low-mass stellar population. Two previous studies (Megeath et al. 1996; Ojha et al. 2004a) report sources with $K$-band excesses indicative of Class I/II PMS stars. It is important to see how the new observations compare and extend the results from previous studies. We find that the derived KLF power-law slope is in remarkable agreement with that of Megeath et al. (1996) and Ojha et al. (2004a) for the W3 IRS 5 cluster region, inspite of a very different sensitivity and resolution. The observed cluster density around W3 IRS 5 (within an apparent radius of $0.27 \mathrm{pc}$ ) is about $\sim 3000$ stars $\mathrm{pc}^{-3}$ for $K<20.5$ as compared to $\sim 2000$ stars pc $^{-3}$ for $K<17.5$ (Ojha et al. 2004a). This high density of stars in W3 Main may be a result of the youth of the cluster and the enormous mass of molecular gas available in the W3 Main molecular core (Megeath et al. 1996). Megeath et al. (1996) found that no more than $30 \%$ of the NIR sources were Class I in the W3 Main region. The present deeper NIR survey also indicates that about $34 \%$ NIR excess sources are Class I. This implies that most of the PMS stars in W3 Main are Class III, as in most other young stellar clusters observed with Chandra, and the age of the low-mass population is > 0.5 Myr (Feigelson \& Townsley, 2008). Ojha et al. (2004a) estimated that the lowest mass limit of Class II and Class I candidates in their observations was $0.1 M_{\odot}$, whereas the high resolution deep imaging presented in this paper is essential for the census of brown dwarf IMF in star forming regions.

\section{Conclusions}

We have successfully conducted the deepest NIR imaging survey of the W3 Main starforming region including IRS 5 with the highest spatial resolution in the star-forming regions thus far. Our main results are summarized as follows:

1. Based on the $J-H$ versus $H-K$ diagram, approximately $167 \mathrm{YSO}$ candidates (Classes I and II) have been identified in a region of $\sim 1^{\prime} .6 \times 1^{\prime} .6$, with $10 \sigma$ limiting magnitude more than 20 in the JHK bands.

2. The KLF of the W3 Main region around IRS 5 shows a power-law slope of $\alpha=0.14 \pm 0.03$, which is in good agreement with the results of Megeath et al. (1996) and Ojha et al. (2004a).

3. We use the presently available theoretical mass-luminosity relation to convert the $J$-band luminosity function into mass function. There appears to be a substantial substellar population of YSOs based on the mass estimates on the basis of this mass - dereddened $J$-band luminosity relationship. 
4. The distribution of bright and faint young stellar candidates versus radial distance is suggestive of mass segregation effects. Extreme youth and estimated dynamical relaxation time indicate that the observed mass segregation in the W3 Main may be the imprint of star formation process.

5. According to our results, it is unlikely that the mass function shows the presence of cutoff and a sharp turnover around the substellar limit, at least at the hydrogen-burning limit.

6. If the center of the W3 IRS 5 cluster is a nascent Trapezium system (Megeath et al. 2005) then the number ratio of BDs and hydrogen-burning stars in the W3 Main is probably higher than that in the Trapezium cluster.

7. If confirmed, these BDs will be the lowest mass members of the W3 IRS5 cluster and therefore provide the key clues to a census of very low mass stars and the IMF down down to about 30 Jupiter-mass for the first time in distant massive star forming region.

We thank the anonymous referee for a critical reading of the paper and several useful comments and suggestions, which greatly improved the scientific content of the paper. This research made use of data collected at Subaru Telescope, which is operated by the National Astronomical Observatory of Japan. We are grateful to the Subaru Telescope staff for their support. We thank T. Sakai for providing us with the FITS image of his CO molecular line observation of W3 Main. We thank Annie Robin for letting us use her model of stellar population synthesis. D. K. O. was supported by the Japan Society for the Promotion of Science through a fellowship, during which most of this work was done.

\section{A. Selected Notable Features}

In Figure 15 we present selected areas of the W3 Main star-forming region in the deepest NIR images so far that are of noteworthy interest.

1. Figure 15a shows the resolved high-mass binary protostellar system W3 IRS 5. A compact nebula is seen between the NIR 1 and NIR 2. The nebula may result from the collision of outflows from NIR 1 and/or NIR 2 (Megeath et al. 2005). We see a dense cluster of embedded stars around IRS 5, a number of these red stars are presumably embedded in the molecular core (Fig. 5).

The previous mid-infrared (MIR) images in fact show the presence of 4 sources of which MIR 1, 2, and 3 are described in van der Tak, Dianchi, \& Tuthill (2005). If we compare 
their $4.7 \mu \mathrm{m}$ image with our $K$-band Image (Fig. 15a), it appears that MIR 2 is in fact a double source and corresponds well with NIR 1 and NIR 2, respectively. But if we compare with longer wavelength $(>9.9 \mu \mathrm{m})$ images, the separation between MIR 2 and MIR 1 gets slightly $\left(0^{\prime \prime} .1-0^{\prime \prime} .2\right)$ shorter (in fact their $9.9 \mu \mathrm{m}$ MIR 2 peak is extended), and MIR 2 does not exactly coincide with NIR 2 but within the "bridge" of nebulosity.

The radio source C or K5 (Claussen et al. 1994; van der Tak, Dianchi, \& Tuthilli, 2005) appears to coincide with the nebula associated with NIR 1 to the west, whereas NIR 5 is

marginally associated with an unnamed radio source of Claussen et al. (1994). There is no NIR ounterpart to radio source a (Claussen et al. 1994).

There is a funny shaped red nebula to the northwest of NIR 1 (Figs. 2 and 15a). This is apparently connected with NIR 1. Interestingly, the nebulosity around this nebula has not been mentioned earlier. Its nature is unknown and no radio source is associated with this nebula (Claussen et al. 1994). However, the direction of the extension of this red nebula is consistent with the large-scale outflow direction (Fig. A1 in Gibb et al. 2007). Thus, this nebula could be a reflection nebula associated with the outflow. The red-shifted component (to southeast) might be invisible even at $K$-band due to much heavier extinction.

2. Figure $15 \mathrm{~b}$ shows the nebulosity associated with the compact H II region W3 B excited by a zero-age main-sequence O-type star IRS 3a. Equally noticeable in Figure 15b are the dark lanes which bisect the nebula. Prominent ones are on the northeast side of the ionized gas and thus are regions of increased extinction comprising ambient molecular gas that has not yet been ionized by the UV radiation field. The appearance of this nebula (Fig. 15b) is very similar to the famous Trifid Nebula (M 20, NGC 6514) in Sagittarius excited by an O star.

3. Dark filamentary lanes are seen (Fig. 15c), with irregular shapes extending throughout the whole W3 Main region. They are associated with the dense molecular gas (Fig. 5). An infrared source (detected only in the $H$ and $K$-bands) marked by an arrow with large color excess $(H-K=4.48)$ is located inside the dark lanes. This is most probably a young star in its earliest evolutionary phase.

4. We detect a faint nebulosity around the ultracompact H II region W3 E (Fig. 15d). Dark edge adjacent to the bright nebulosity is particularly striking.

\section{REFERENCES}

Baraffe, I., Chabrier, G., Allard, F., \& Hauschildt, P. H. 1998, A\&A, 337, 403 
Baraffe, I., Chabrier, G., Barman, T. S., Allard, F., \& Hauschildt, P. H. 2003, A\&A, 402, 701

Bessell, M. S., \& Brett, J. M. 1988, PASP, 100, 1134

Claussen, M. J., Gaume, R. A., Johnston, K. J., \& Wilson, T. L. 1994, ApJ, 424, L41.

Cohen, J. G., Persson, S. E., Elias, J. H., \& Frogel, J. A. 1981, ApJ, 249, 481

Comeron, F., Rieke, G. H., Burrows, A., \& Rieke, M. J. 1993, ApJ, 416, 185

DeGioia Eastwood, K., Throop, H., Walker, G., \& Cudworth, K. M. 2001, ApJ, 549, 578

Feigelson, E. D., \& Townsley, L. K. 2008, ApJ, 673, 354

Gardner, J. P., Cowie, L. L., \& Wainscoat, R. J. 1993, ApJ, 415, L9

Gibb, A. G., Davis, C. J., \& Moore, T. J. T. 2007, MNRAS, 382, 1213

Haisch, K. E., Lada, E. A., \& Lada, C. J. 2000, AJ, 120, 1396

Haisch, K. E., Lada, E. A., \& Lada, C. J. 2001, AJ, 121, 2065

Hawarden, T. G., Leggett, S. K., Letawsky, M. B., Ballantyne, D. R., \& Casali, M. M. 2001, MNRAS, 325, 563

Herbst, W., Miller, D. P. 1982, AJ, 87, 1478

Hillenbrand, L. A., Strom, S. E., Vrba, F. J., \& Keene, J. 1992, ApJ, 397, 613

Hillenbrand, L. A. 1997, AJ, 113, 1733

Hillenbrand, L. A., \& Carpenter, J. M. 2000, AJ, 540, 236

Jose, J., et al. 2008, MNRAS, 384, 1675

Kaifu, N., et al. 2000, PASJ, 52, 1

Kenyon, S. J., \& Hartmann, L. 1995, ApJS, 101, 117

Koornneef, J. 1983, A\&A, 128, 84

Kroupa, P. 2001, MNRAS, 322, 231

Kroupa, P. 2002, Science, 295, 82

Lada, C. J., Young, E. T., \& Greene, T. P. 1993, ApJ, 408, 471 
Lada, C. J., et al. 2000, AJ, 120, 3162

Lada, C. J., \& Lada, E. A. 2003, ARA\&A, 41, 5

Lada, E. A., Evans, N. J., Depoy, D. L., \& Gatley, I. 1991, ApJ, 371, 171

Liu, M. C., Najita, J., \& Tokunaga, A. T. 2003, ApJ, 585, 372

Lucas, P. W., \& Roche, P. F. 2000, MNRAS, 314, 858

Luhman, K. L., \& Rieke, G. H. 1999, ApJ, 525, 440

Luhman, K. L., et al. 2000, ApJ, 540, 1016

Luhman, K. L., Joergens, V., Lada, C., Muzerolle, J., Pascucci, I., \& White, R. 2007, Protostars and Planets V, B. Reipurth, D. Jewitt, and K. Keil (eds.), University of Arizona Press, Tucson, p.443-457

Maihara, T., et al. 2001, PASJ, 53, 25

Mathieu, R. D. 1985, Int. Astron. Union Symp., 113, 427

Mathieu, R. D., \& Latham, D. W. 1986, AJ, 92, 1364

Mathis, J. S. 1990, ARA\&A, 28, 37

McLeod, B. A., Bernstein, G. M., Rieke, M. J., Tollestrup, E. V., \& Fazio, G. G. 1995, ApJS, 96, 117

McNamara, B. J., \& Sekiguchi K. 1986, ApJ, 310, 613

Megeath, S. T., Herter, T., Beichman, C., Gautier, N., Hester, J. J., Rayner, J., \& Shupe, D. 1996, A\&A, 307, 775

Megeath, S. T., Wilson, T. L., \& Corbin, M. R. 2005, ApJ, 622, L141

Meyer, M., Calvet, N., \& Hillenbrand, L. A. 1997, AJ, 114, 288

Motohara, K., et al. 2002, PASJ, 54, 315

Muench, A. A., Alves, J., Lada, C. J., \& Lada, E. A. 2001, ApJ, 558, L51

Muench, A. A., Lada, E. A., Lada, C. J., \& Alves, J. 2002, ApJ, 573, 366

Muench, A. A., et al. 2003, AJ, 125, 2029 
Nakajima, Y., Tamura, M., Oasa, Y., \& Nakajima, T. 2000, AJ, 119, 873

Oasa, Y., Tamura, M., \& Sugitani, K. 1999, ApJ, 526, 336

Oasa, Y., et al. 2006, AJ, 131, 1608

Ojha, D. K., et al. 2004a, ApJ, 608, 797

Ojha, D. K., et al. 2004b, ApJ, 616, 1042

Oliveira, J. M., Jeffries, R. D., van Loon, J. Th., Littlefair, S. P., \& Naylor, T. 2005, MNRAS, 358, L21

Palla, F., \& Stahler, S. 1999, ApJ, 525, 772

Pandey, A. K., Upadhyay, K., Ogura, K., Sagar, R., Mohan, V., Mito, H., Bhatt, H. C., \& Bhatt, B. C. 2005, MNRAS, 358, 1290

Pandey, A. K., Sharma, S., Ogura, K., Ojha, D. K., Chen, W. P., Bhatt, B. C., \& Ghosh, S. K. 2008, MNRAS, 383, 1241

Robin, A. C., Reylé, C., Derriére, S., \& Picaud, S. 2003, A\&A, 409, 523

Salpeter, E. E. 1955, ApJ, 121, 161

Saracco, P., D’Odorico, S., Moorwood, A., Buzzoni, A., Cuby, J. G., \& Lidman, C. 1999, A\&A, 349, 751

Sharma, S., Pandey, A. K., Ojha, D. K., Chen, W. P., Ghosh, S. K., Bhatt, B. C., Maheswar, G., \& Sagar, R. 2007, MNRAS, 380, 1141

Spitzer, L., Jr, \& Hart, M. H. 1971, ApJ, 164, 399

Stetson, P. B. 1987, PASP, 99, 191

Sugitani, K., et al. 2002, ApJ, 565, L25

Tamura, M., Itoh, Y., Oasa, Y., \& Nakajima, T. 1998, Science, 282, 1095

Teixeira, P. S., Fernandes, S. R., Alves, J. F., Correia, J. C., Santos, F. D., Lada, E. A., \& Lada, C. J. 2004, A\&A, 413, L1

van der Tak, F. F. S., Tuthill, P. G., \& Danchi, W. C. 2005, A\&A, 431, 993

Zinnecker, H., McCaughrean, M. J., \& Wilking, B. A. 1993, In: Protostars and Planets III, p. $429-495$ 
This preprint was prepared with the AAS LATEX macros v5.2. 
Table 1: NIR sources around W3 IRS 5

\begin{tabular}{ccccc}
\hline \hline Source & $\begin{array}{c}\text { R.A. } \\
\text { (J2000.0) }\end{array}$ & $\begin{array}{c}\text { Decl. } \\
(\mathrm{J} 2000.0)\end{array}$ & $\begin{array}{c}H S T \text { F222 } M^{\dagger} \\
(\mathrm{mag})\end{array}$ & $\begin{array}{c}\text { Subaru } K \text {-band } \\
(\mathrm{mag})\end{array}$ \\
\hline NIR 3 & 22540.73 & +620549.9 & $17.94 \pm 0.10$ & $17.38 \pm 0.04$ \\
NIR 4 & 22540.90 & +620551.7 & $16.20 \pm 0.07$ & $16.09 \pm 0.07$ \\
NIR 5 & 22541.04 & +620552.1 & $17.94 \pm 0.10$ & $17.90 \pm 0.07$ \\
\hline
\end{tabular}

${ }^{\dagger}$ Megeath et al. (2005) 

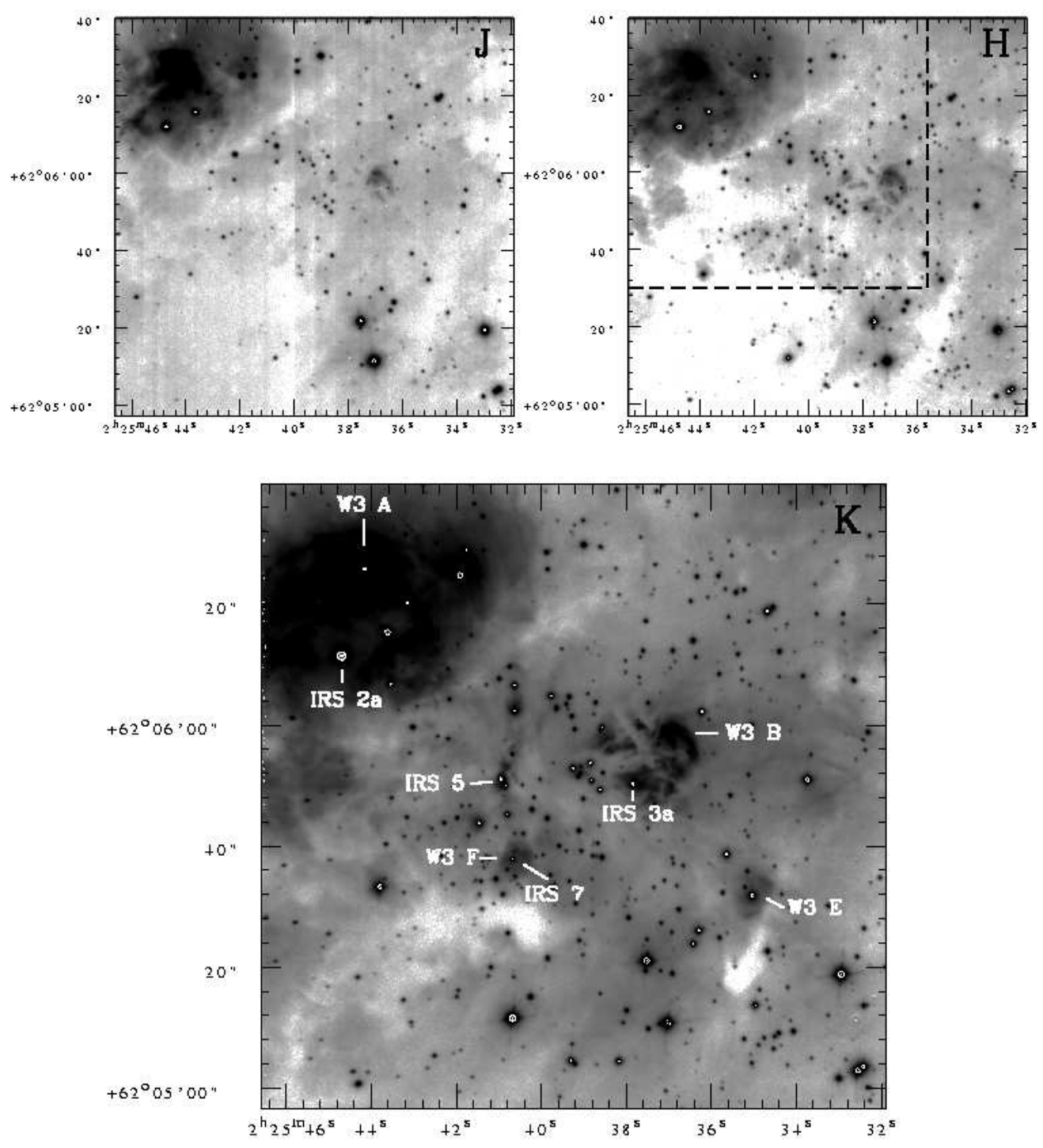

Fig. 1.- $J_{-}, H_{-}$, and $K$-band images of the W3 Main star-forming region displayed in a logarithmic intensity scale. The box marked with the dashed lines in the $H$-band image shows the cluster region around W3 IRS 5 and diffuse region to the northeast. The locations of the individual H II and ultracompact H II regions and the embedded IR sources are marked in the $K$-band image. North is up and east is to the left. The abscissa and the ordinate are in J2000.0 epoch. 


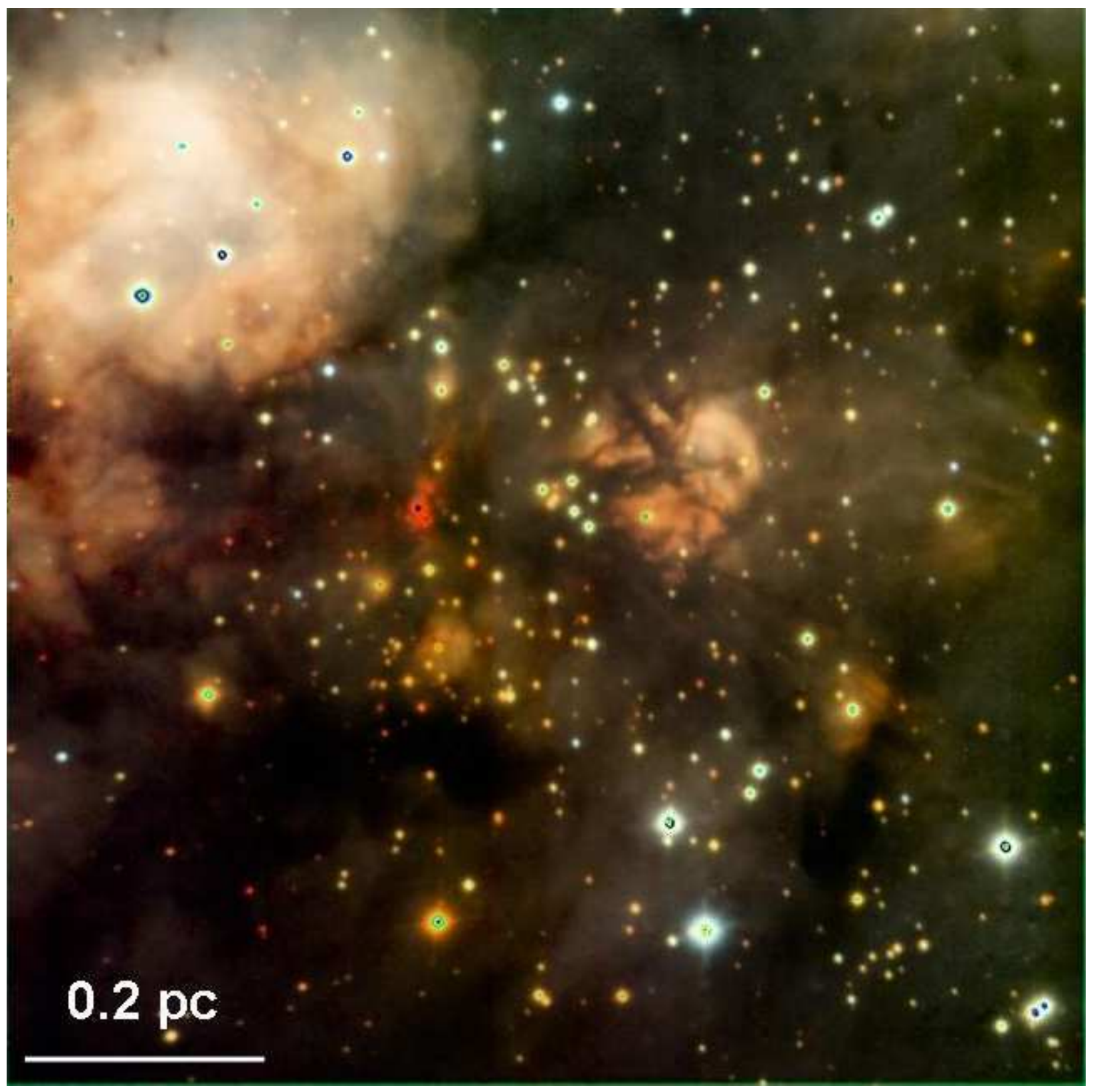

Fig. 2.- JHK composite image of the W3 Main star-forming region ( $J$ : blue; $H$ : green; $K$ : red) obtained with the CISCO mounted on the Subaru $8.2 \mathrm{~m}$ telescope. The FOV is $\sim 1^{\prime} .8$ $\times 1^{\prime} .8$. North is up, and east is to the left. 

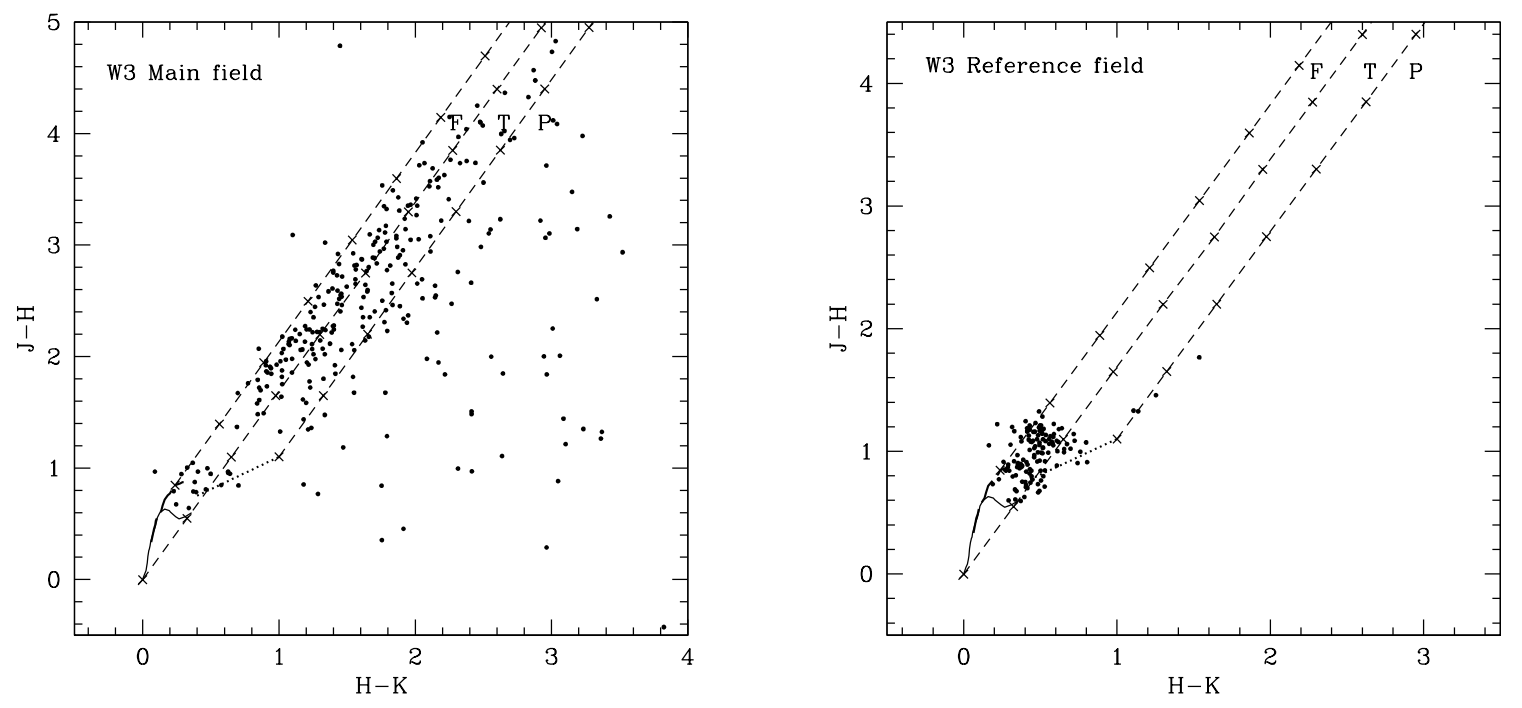

Fig. 3.- CC diagram of the W3 Main star-forming region (left) and the reference field (right) for the sources detected in $J H K$ bands with photometric errors less than 0.1 mag. The sequences for field dwarfs (solid curve) and giants (thick dashed curve) are from Bessell \& Brett (1988). The dotted line represents the locus of T Tauri stars (Meyer et al. 1997). Dashed straight lines represent the reddening vectors (Cohen et al. 1981). The crosses on the dashed lines are separated by $A_{V}=5 \mathrm{mag}$. The plot is classified into three regions, namely, F, T, and P for different classes of sources (see text for details). 

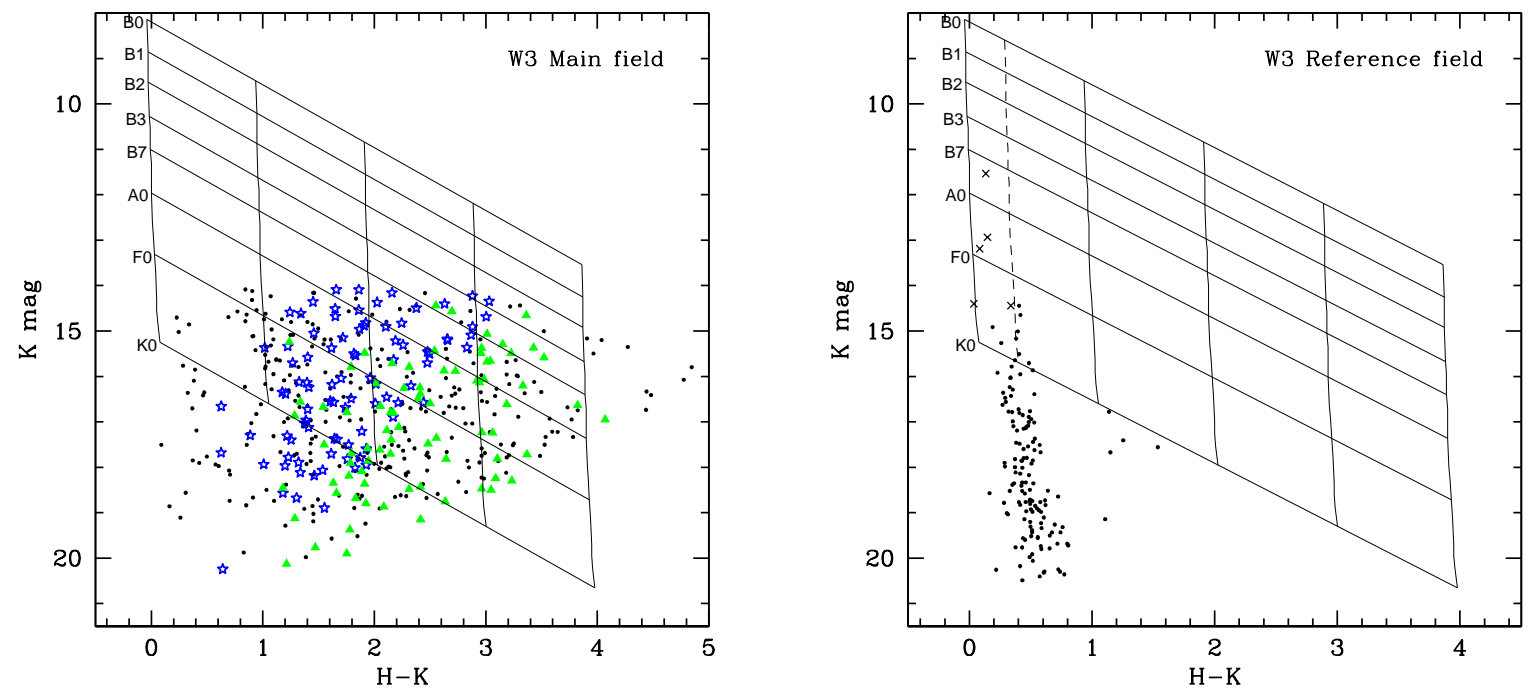

Fig. 4. $-H-K / K \mathrm{CM}$ diagram for the sources detected in $H$ and $K$ bands with photometric errors less than $0.1 \mathrm{mag}$ in the W3 Main star-forming region (left) and the reference field (right). Stars and filled triangles represent the YSOs identified from the regions "T" and "P" in Fig. 3, respectively. The vertical solid lines from left to right indicate the mainsequence track at $1.83 \mathrm{kpc}$ reddened by $A_{V}=0,15,30,45$, and $60 \mathrm{mag}$, respectively. The intrinsic colors are taken from Koornneef (1983). The dashed line in the reference field (right) shows the main-sequence track reddened by $A_{V}=5 \mathrm{mag}$, and the cross symbols denote 2MASS sources brigher than $K=14.5 \mathrm{mag}$, which are saturated in our observations. Slanting horizontal lines identify the reddening vectors (Cohen et al. 1981). 


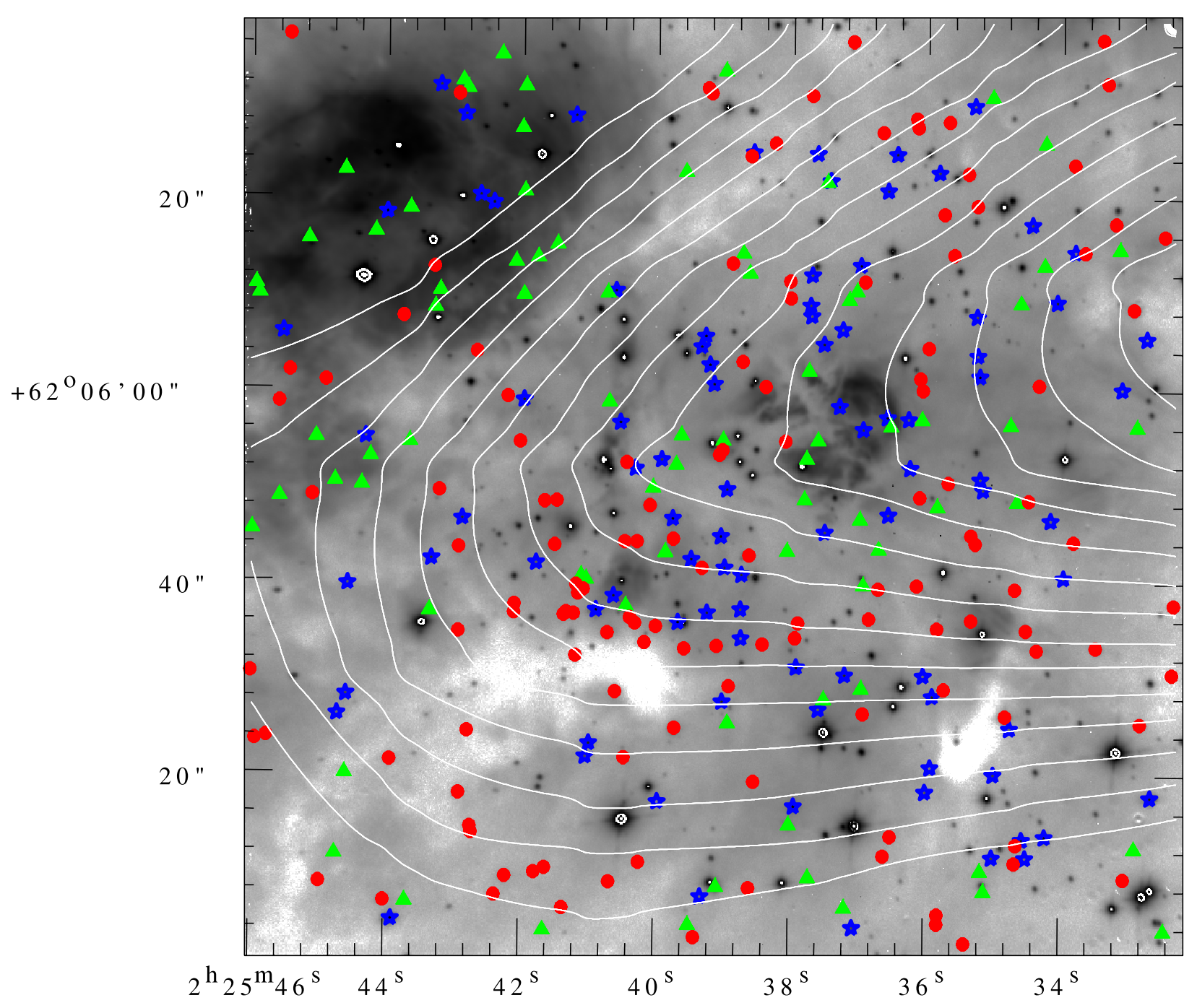

Fig. 5.- Spatial distribution of the YSO color-excess candidates superposed on the $K$-band image with a logarithmic intensity scale. The white contours show the integrated emission of the ${ }^{12} \mathrm{CO}$ line. The $\mathrm{CO}$ contours start at $21 \mathrm{~K} \mathrm{~km} \mathrm{~s}^{-1}$ and increase in steps of $2 \mathrm{~K} \mathrm{~km} \mathrm{~s}^{-1}$. Blue stars represent $\mathrm{T}$ Tauri and related sources (Class II), green triangles indicate Class I sources, and red circles denote the red sources $(H-K>2)$. The abscissa and the ordinate are in J2000.0 epoch. 


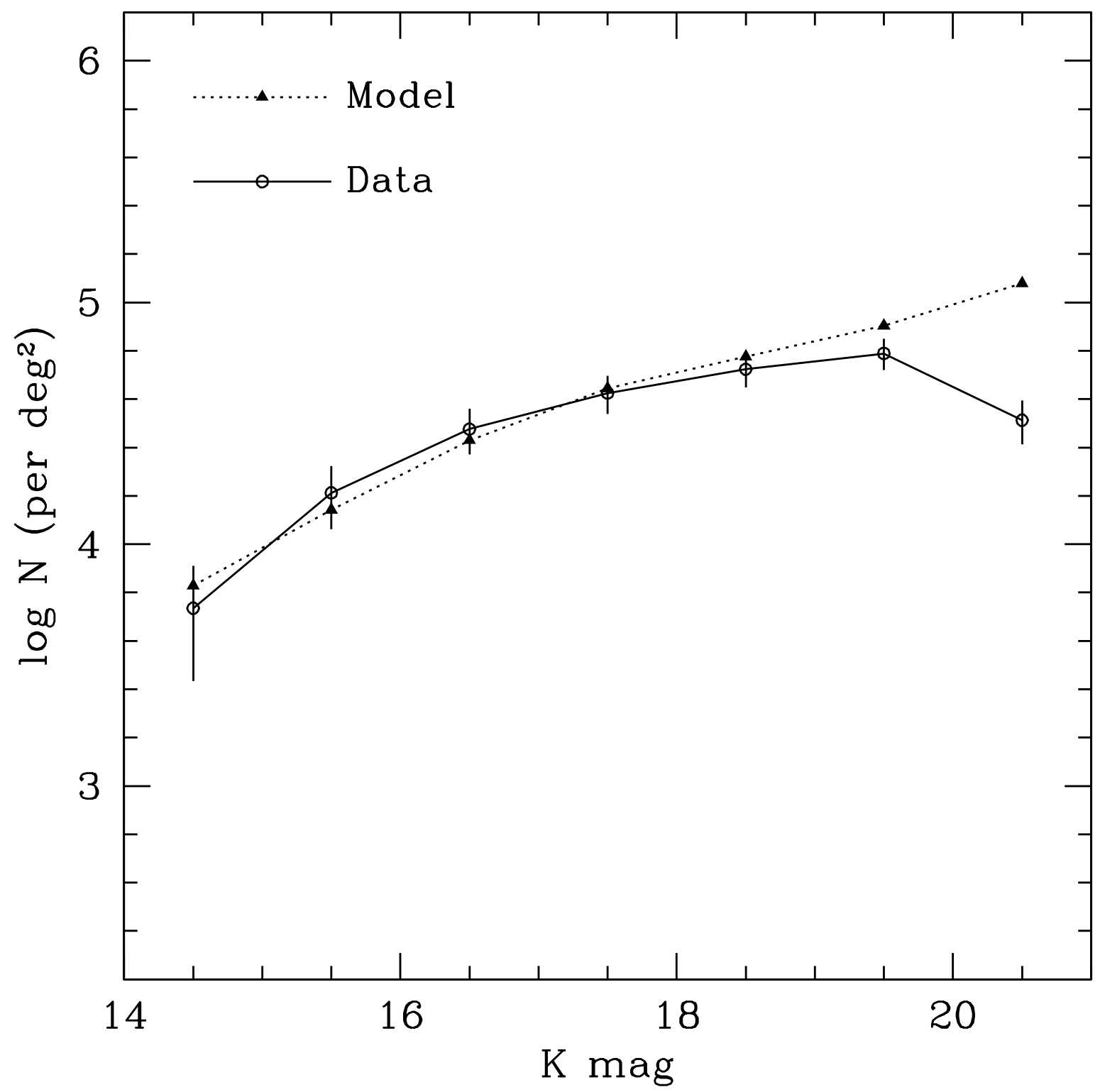

Fig. 6.- Completeness-corrected KLF of the reference field (solid line) is compared with the model predicted KLF where an extinction of $A_{V}=5$ mag is applied to all the model stars (dotted line). The KLF slope ( $\alpha$; see $\S 4.4)$ of the dotted line (model simulation) is $0.20 \pm 0.02$. 


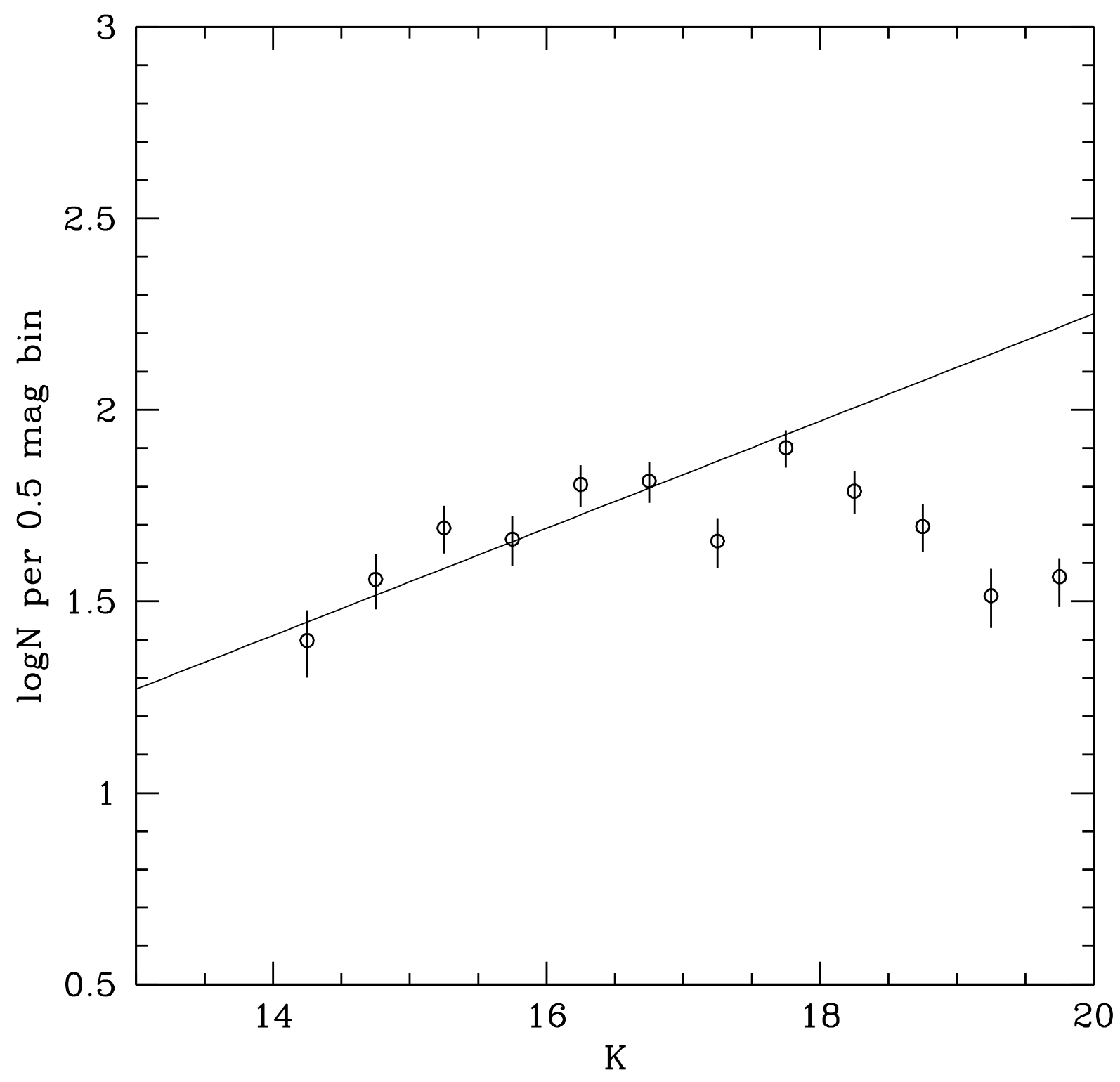

Fig. 7.- Logarithm of the completeness-corrected and field star-subtracted KLF of the W3 Main region. The solid line is the least-square linear fit to the data points. 


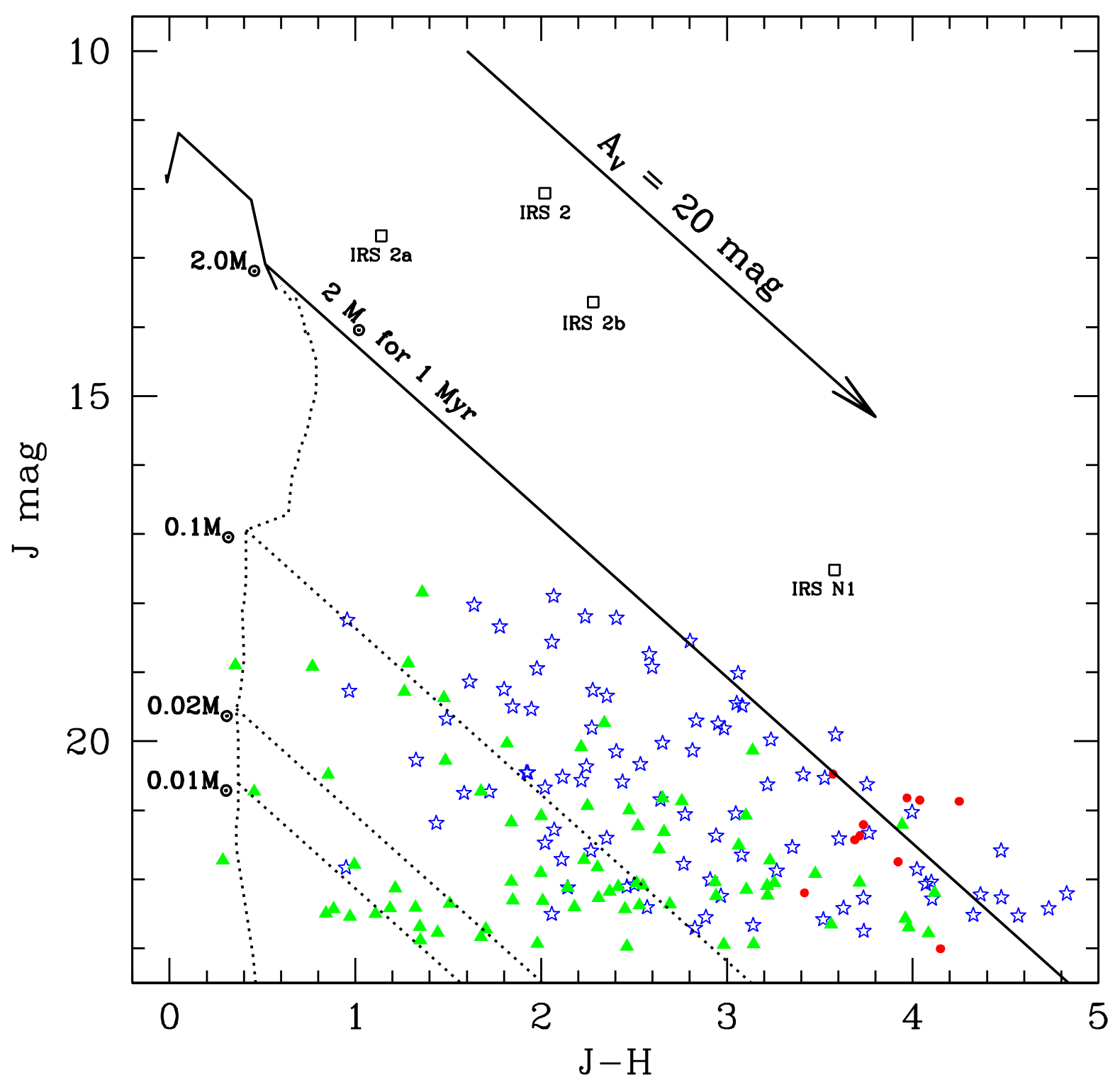

Fig. 8. - CM diagram for the YSO candidates in W3 Main. Class II candidates are indicated by stars, filled triangles represent Class I candidates, and the filled circles show red sources with $H-K>2$ with $J$-band counterparts. The solid and dotted curves denote the loci of 1 Myr old PMS stars; derived from the models of Palla \& Stahler (1999), and Baraffe et al. $(1998 ; 2003)$, respectively. Masses range from 1.4 to $4.0 \mathrm{M}_{\odot}$ and 0.0005 to $1.4 \mathrm{M}_{\odot}$ from bottom to top, for the solid and dotted curves, respectively. Also shown are the positions of known IRS sources (see Table 1 in Ojha et al. 2004a). 

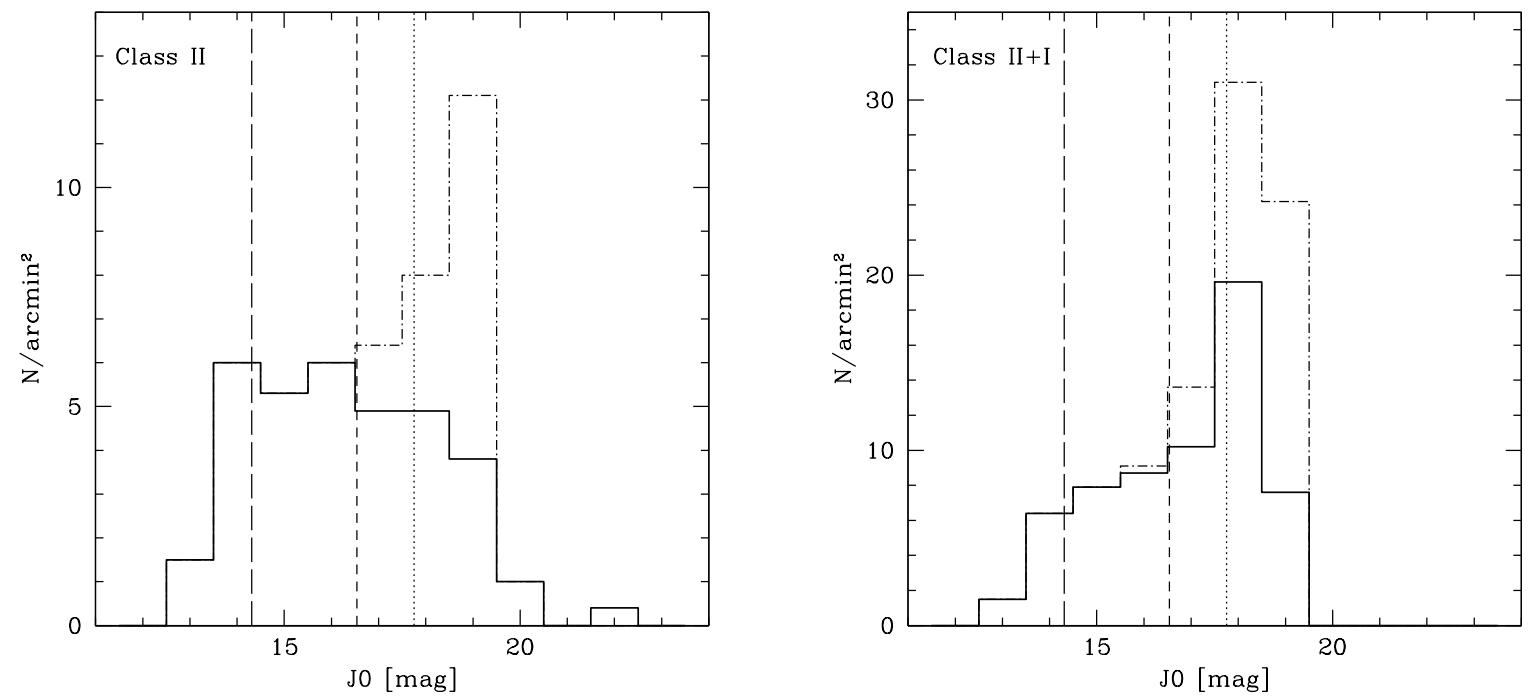

Fig. 9.- Reddening-corrected $J$-band luminosity functions (solid line) of the identified Class II (left) and combined (Class II + I) objects (right). The vertical dotted line represents the star-BD boundary at $1 \mathrm{Myr}$, while the short-dashed and long-dashed lines represent $90 \%$ and $100 \%$ completeness limits, respectively. The dashed-dotted line indicates the JLF corrected for the completeness in the respective $J$-band magnitude bins. 


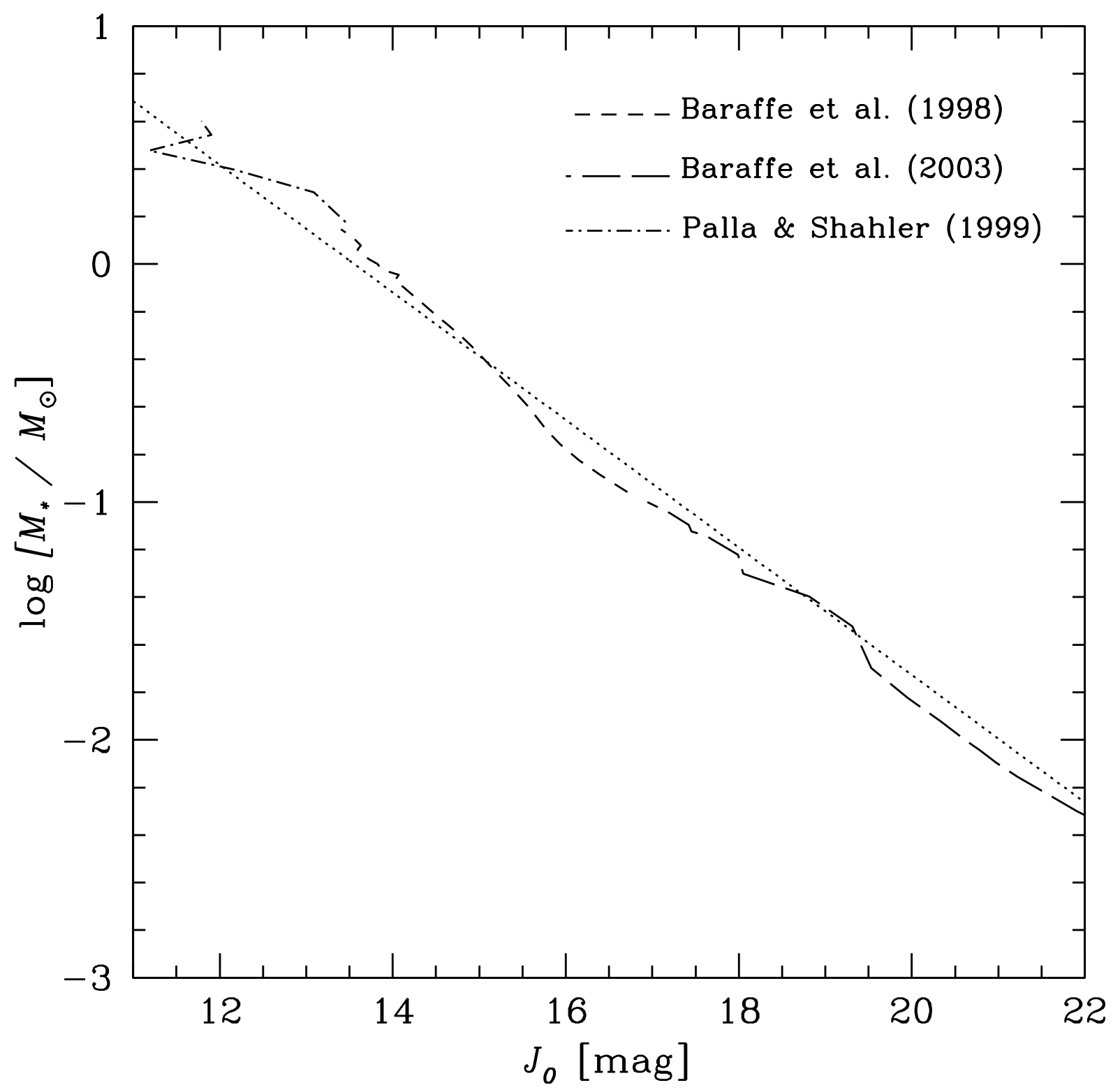

Fig. 10.- J-band luminosity - mass relationship for low-mass YSOs with an age of $1 \mathrm{Myr}$ based on the evolutionary models of Baraffe et al. (1998; 2003), and Palla \& Stahler (1999). The dotted line shows a least-square linear fit to the mass range $0.0005<M / M_{\odot}<4.0$. 

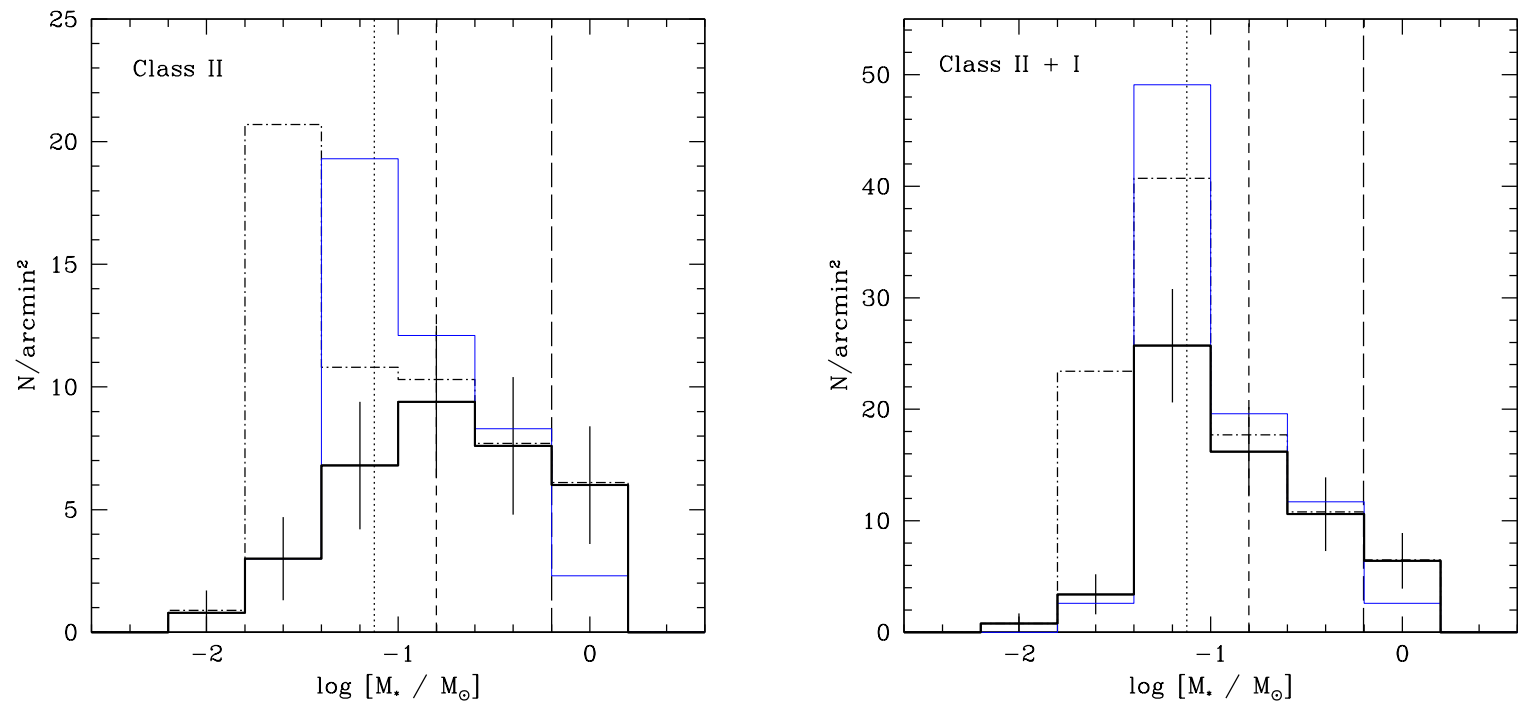

Fig. 11. - Mass functions (with $\pm \sqrt{N}$ error bars) for the identified Class II (left) and combined (Class II + I) objects (right), based on the evolutionary models of Baraffe et al. (1998; 2003), and Palla \& Stahler (1999), for the age assumption of 1 Myr. The dasheddotted line shows the MF corrected for the completeness in the respective mass bins. The dotted, short-dashed, and long-dashed lines are the same as in Figure 9. The thin continuous line indicates the completeness-corrected MF derived using reddening-corrected JLF with an average extinction subtraction (see $\S 4.6$ ). 

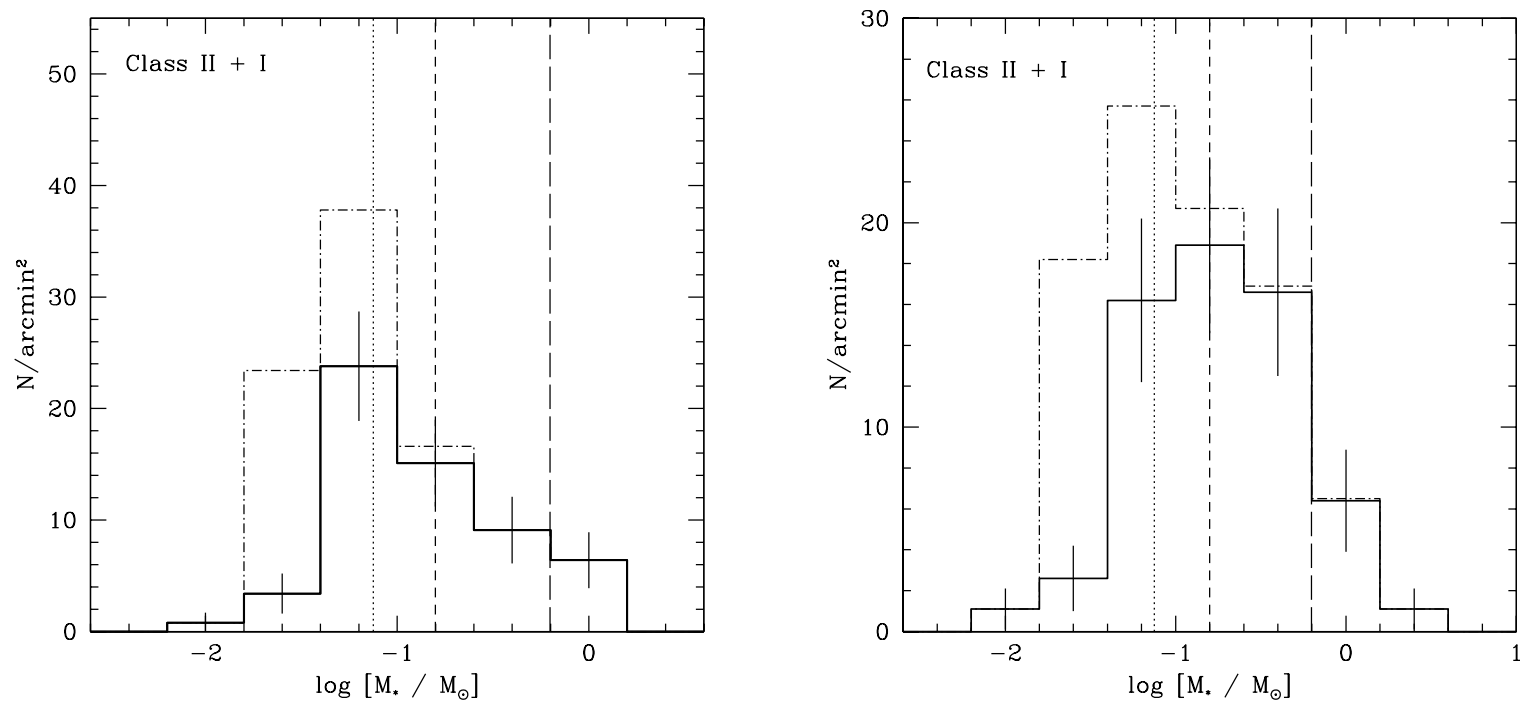

Fig. 12.- (left) The composite MF (with $\pm \sqrt{N}$ error bars) for Class II and Class I sources (solid line), where sources in the "P" region that fall below $J-H=1.0$ are not included. (right) The MF for all the sources in "T" (Class II) and "P" (Class I) regions (solid line). The sources are dereddened by using the extinction map (see text for details). The dasheddotted line in both the diagrams indicates the MF corrected for the completeness in the respective mass bins. The dotted, short-dashed, and long-dashed lines are the same as in Figure 9 . 


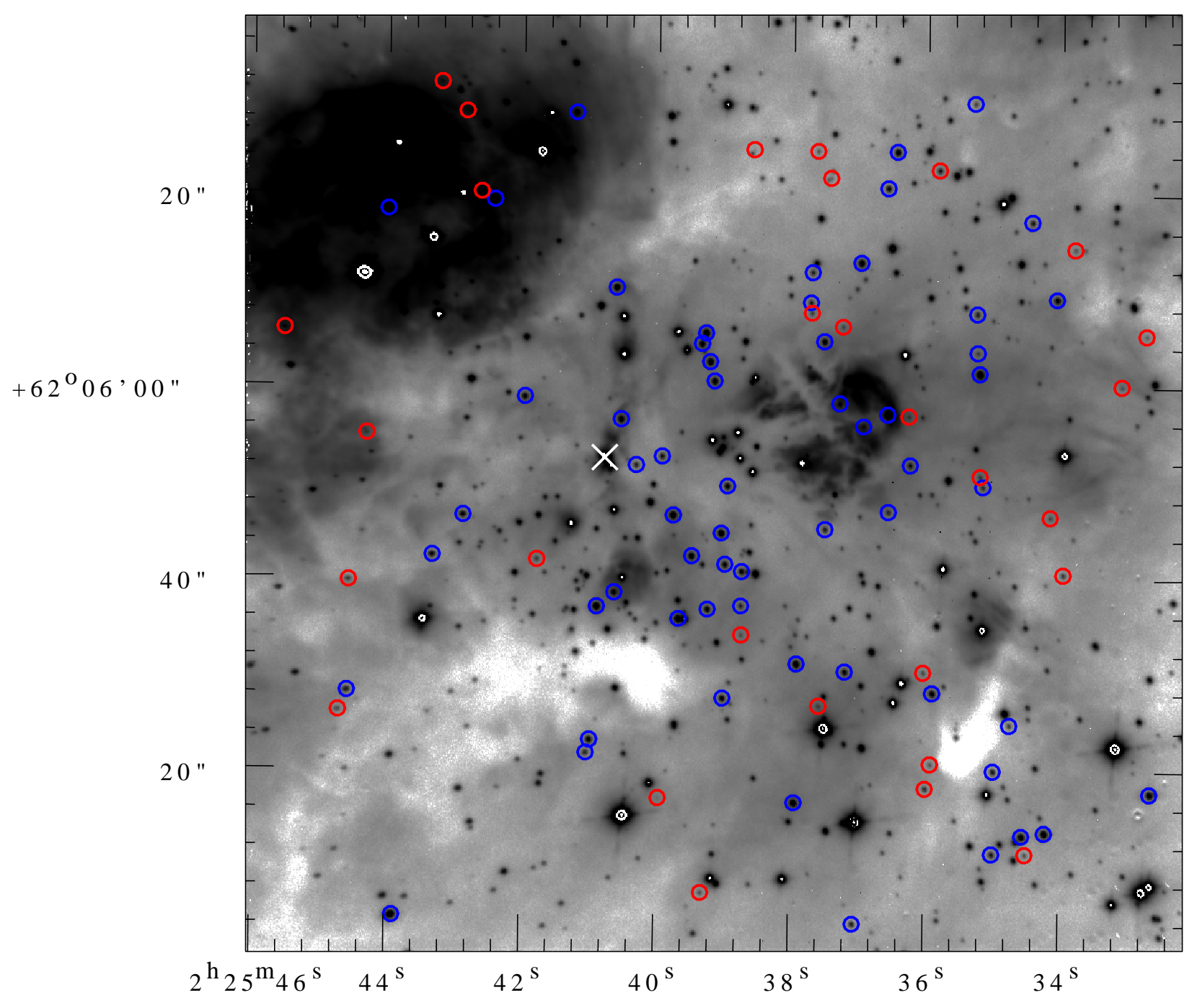

Fig. 13. - Spatial distribution of the Class II sources in two magnitude intervals (blue: $J_{0}$ $<17.25 \mathrm{mag}$; red: $\left.J_{0}>17.25 \mathrm{mag}\right)$ superposed on the $K$-band image. The center position of W3 IRS 5 is marked with a cross. 

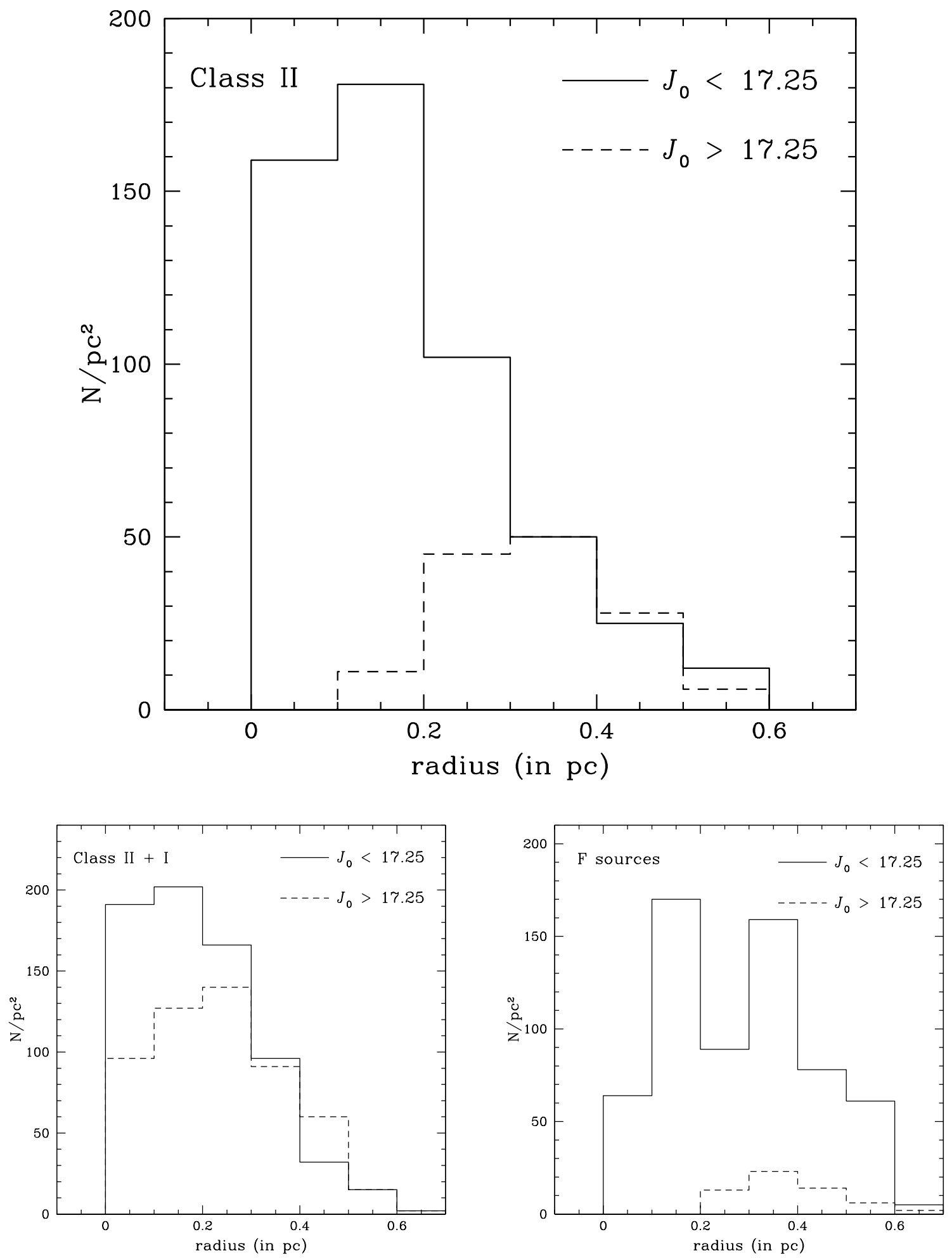

Fig. 14. - The radial distribution of Class II (top), composite (Class II and I) (bottom left), and " $\mathrm{F}$ " sources (bottom right) in two dereddend $J$-band magnitude intervals. The radial distance is calculated with respect to the positions of W3 IRS 5. 


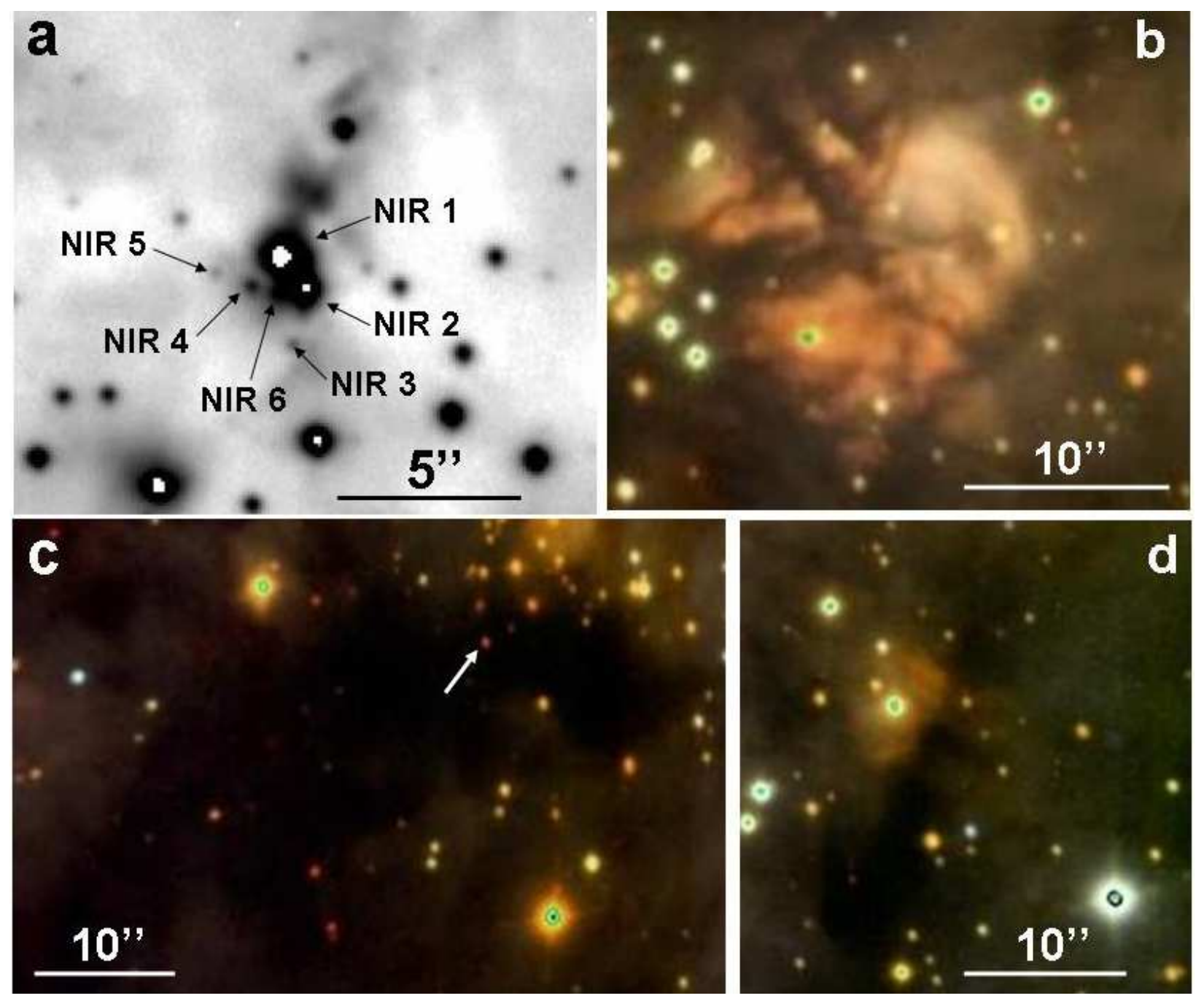

Fig. 15. - Enlarged view of the $K$-band and color composite images of selected areas (see Figs. 1, 2, and the Appendix). (a) Section of the $K$-band image around W3 IRS 5 region and the neighboring red sources and nebulosities. In its immediate vicinity extremely red infrared sources, are located (marked by NIR 3, NIR 4, NIR 5, and NIR 6; see §4.2). (b) Fluffy diffuse nebula W3 B, probably illuminated by the bright star(s) located southeast of this feature. (c) Dark filamentary lanes with irregular shapes seen throughout the whole W3 Main star-forming region. An infrared source marked by an arrow, with large color excess, is located inside one of them. (d) A faint nebulosity detected around the ultracompact H II region $\mathrm{W} 3 \mathrm{E}$. 